\title{
INFRARED SPECTRAL ENERGY DISTRIBUTION DECOMPOSITION OF WISE-SELECTED, HYPERLUMINOUS HOT DUST-OBSCURED GALAXIES
}

\author{
Lulu Fan (范璐璐 $)^{1,2}$, YUnKun Han (韩云坤 $)^{3}$, Robert NikUtTa ${ }^{4}$, Guillaume DrouarT ${ }^{2}$, ANd Kirsten K. KNUdSEN ${ }^{2}$ \\ ${ }^{1}$ Shandong Provincial Key Lab of Optical Astronomy and Solar-Terrestrial Environment, Institute of Space Science, \\ Shandong University, Weihai, 264209, China; 1lfan@sdu.edu.cn \\ ${ }^{2}$ Department of Earth and Space Sciences, Chalmers University of Technology, Onsala Space Observatory, SE-439 92 Onsala, Sweden \\ ${ }^{3}$ Yunnan Observatories, Chinese Academy of Sciences, Kunming, 650011, China; hanyk@ynao.ac.cn \\ ${ }^{4}$ Instituto de Astrofísica, Facultad de Física, Pontificia Universidad Católica de Chile, 306, Santiago 22, Chile \\ Received 2015 December 30; accepted 2016 March 22; published 2016 May 26
}

\begin{abstract}
We utilize a Bayesian approach to fit the observed mid-IR-to-submillimeter/millimeter spectral energy distributions (SEDs) of 22 WISE-selected and submillimeter-detected, hyperluminous hot dust-obscured galaxies (Hot DOGs), with spectroscopic redshift ranging from 1.7 to 4.6. We compare the Bayesian evidence of a torus plusgraybody (Torus+GB) model with that of a torus-only (Torus) model and find that the Torus+GB model has higher Bayesian evidence for all 22 Hot DOGs than the torus-only model, which presents strong evidence in favor of the Torus+GB model. By adopting the Torus+GB model, we decompose the observed IR SEDs of Hot DOGs into torus and cold dust components. The main results are as follows. (1) Hot DOGs in our submillimeter-detected sample are hyperluminous $\left(L_{\mathrm{IR}} \geqslant 10^{13} L_{\odot}\right)$, with torus emission dominating the IR energy output. However, cold dust emission is non-negligible, contributing on average $\sim 24 \%$ of total IR luminosity. (2) Compared to QSO and starburst SED templates, the median SED of Hot DOGs shows the highest luminosity ratio between mid-IR and submillimeter at rest frame, while it is very similar to that of QSOs at $\sim 10-50 \mu \mathrm{m}$, suggesting that the heating sources of Hot DOGs should be buried AGNs. (3) Hot DOGs have high dust temperatures $\left(T_{\text {dust }} \sim 72 \mathrm{~K}\right.$ ) and high IR luminosity of cold dust. The $T_{\text {dust }}-L_{\mathrm{IR}}$ relation of Hot DOGs suggests that the increase in IR luminosity for Hot DOGs is mostly due to the increase of the dust temperature, rather than dust mass. Hot DOGs have lower dust masses than submillimeter galaxies (SMGs) and QSOs within a similar redshift range. Both high IR luminosity of cold dust and relatively low dust mass in Hot DOGs can be expected by their relatively high dust temperatures. (4) Hot DOGs have high dust-covering factors (CFs), which deviate from the previously proposed trend of the dust CF decreasing with increasing bolometric luminosity. Finally, we can reproduce the observed properties in Hot DOGs by employing a physical model of galaxy evolution. This result suggests that Hot DOGs may lie at or close to peaks of both star formation and black hole growth histories, and represent a transit phase during the evolutions of massive galaxies, transforming them from the dusty starburst-dominated phase to the optically bright QSO phase.
\end{abstract}

Key words: galaxies: active - galaxies: evolution - galaxies: formation - galaxies: high-redshift - infrared: galaxies

\section{INTRODUCTION}

In the popular framework of galaxy formation and evolution (e.g., Hopkins et al. 2006, 2008), massive galaxies have been proposed to co-grow with their central supermassive black holes (SMBHs). Intense starbursts are triggered by major gasrich mergers (Barnes \& Hernquist 1992; Hopkins et al. 2008; Wuyts et al. 2010) or violent disk instabilities (VDI; Dekel et al. 2009), which also provide the fuel for central black hole accretion. Host galaxy and SMBH grow coevally, experiencing starburst-dominated, active galaxy nuclei (AGNs)/QSO and starburst composite and AGN-dominated phases, until the AGN feedback is strong enough to expel gas and dust, making star formation and AGN activity itself come to an end on a short timescale, and finally leaving a passively evolved galaxy (Sanders \& Mirabel 1996; Granato et al. 2004; Hopkins et al. 2006, 2008; Alexander \& Hickox 2012). During the intense star formation episode, a significant amount of dust is produced, which plays an important role in shaping the observed spectral energy distribution (SED) of a massive evolving galaxy in different phases. Dust absorbs most of the UV and optical photons and re-emits in the far-infrared (FIR) and submillimeter (submm) wavelengths. Starburst-dominated and AGN-starburst composite systems will therefore appear to be IR luminous, just as those observed populations: ultra- luminous infrared galaxies (ULIRGs; Sanders \& Mirabel 1996), submillimeter galaxies (SMGs; Blain et al. 2002; Chapman et al. 2005; Casey et al. 2014) and dust-obscured galaxies (DOGs; Dey et al. 2008). Studying the IR luminous galaxies at high redshift will help us understand the extreme scenarios in the early phase of massive galaxy evolution.

Recently, Eisenhardt et al. (2012) and Wu et al. (2012) discovered a new population of hyperluminous DOGs using NASA's Wide-field Infrared Survey Explorer (WISE ) mission (Wright et al. 2010). They selected objects using the so-called "W1W2-dropout" method. They selected those objects that are prominent in the WISE12 $\mu \mathrm{m}$ (W3) or $22 \mu \mathrm{m}$ (W4) bands, and that are faint or undetected in the $3.4 \mu \mathrm{m}$ (W1) and $4.6 \mu \mathrm{m}$ (W2) band. These objects are rare. In total, about 1000 such objects have been identified in all of the sky (Eisenhardt et al. 2012). Among them, about 150 objects have had spectroscopic follow-up and have been found to mostly be at high redshift, with redshift ranging from 1 to 4 (Wu et al. 2012; Tsai et al. 2015).

In order to understand the dust properties and calculate the total luminosities of these unusual galaxies, continuum measurements at longer wavelengths are crucial. Wu et al. (2012) observed 14 W1W2-dropout galaxies at $z>1.7$ with the Caltech Submillimeter Observatory (CSO) SHARC-II at $350-850 \mu \mathrm{m}$, with 9 detections, and observed 18 with the CSO 
Bolocam at $1.1 \mathrm{~mm}$, with 5 detections. Jones et al. (2014) used the SCUBA-2 (Submillimetre Common-User Bolometer Array) $850 \mu \mathrm{m}$ band to observe 10 dusty, luminous galaxies at $z \sim 1.7-4.6$, with 6 detections. Combined with WISE photometry with Herschel PACS and SPIRE data (Tsai et al. 2015), the IR SEDs of these objects have been found to be very different from other known populations. Their SEDs have a high mid-IR to submm luminosity ratio, which suggests that their IR luminosities are dominated by emission from hot dust. Therefore, $\mathrm{Wu}$ et al. (2012) referred to these galaxies as hot, dust-obscured galaxies, or Hot DOGs. They are also hyperluminous: most have luminosities well over $10^{13} L_{\odot}$, and some exceed $10^{14} L_{\odot}$, comparable to the most luminous quasars known (Assef et al. 2015a; Tsai et al. 2015). The hot dust temperature and extremely high luminosity indicate that these objects are likely heavily obscured quasars. The recent X-ray data of several Hot DOGs observed by XMM-Newton, Chandra, and NuSTAR are consistent with the scenario of them being hyperluminous, highly obscured AGNs (Stern et al. 2014; Assef et al. 2015b; Piconcelli et al. 2015).

Besides the heavily obscured QSOs in the center of Hot DOGs, they are also likely to host intense star formation, suggested by the submillimeter/millimeter $(\mathrm{mm})$ detections (Jones et al. 2014; Wu et al. 2014). Thus, Hot DOGs may represent an AGN-starburst composite system, experiencing a transit phase from a dust-obscured phase to an unobscured QSO phase. The relative contributions of AGNs and starbursts, which have not been well investigated in previous works, can be analyzed based on the detailed IR SED decomposition. Different IR SED decomposition methods have been recently carried out to analyze ULIRGs, high- $z$ radio galaxies, and QSOs in the literature (Mullaney et al. 2011; Han \& Han 2012; Drouart et al. 2014; Leipski et al. 2014; Ma \& Yan 2015; Xu et al. 2015).

Here we construct complete mid-IR to submm/mm SEDs of a submm-detected Hot DOG sample with spectroscopic redshift and use a Bayesian approach to decompose the different dust components, separating contributions from the AGN and the starburst. In Section 2, we describe the sample selection, the photometry of Herschel observations and the compilation of mid-IR to submm/mm SEDs. In Section 3, we present our Bayesian approach for IR SED decomposition. Results and discussions are described in Sections 4 and 5, respectively. We summarize our main results in Section 6 . Throughout this work we assume a flat $\Lambda$ CDM cosmology with $H_{0}=70 \mathrm{~km} \mathrm{~s}^{-1}, \Omega_{M}=0.3$, and $\Omega_{\Lambda}=0.7$.

\section{DATA}

\subsection{Sample}

The Hot DOGs studied here are selected from the WISE AllSky Source catalog 5 , which provides point-spread functionfitting magnitudes and uncertainties in the Vega system (Cutri et al. 2013). The detailed selection criteria are $\mathrm{W} 1>17.4(<34$ $\mu \mathrm{Jy})$ and either $\mathrm{W} 4<7.7$ ( $>6.9 \mathrm{mJy})$ and $\mathrm{W} 2-\mathrm{W} 4>8.2$, or $\mathrm{W} 3<10.6$ (>1.7 mJy) and W2 - W3 $>5.3$ (Eisenhardt et al. 2012; Wu et al. 2012). With several additional constraints, the resulting sample contains 934 objects over approximately 32,000 $\mathrm{deg}^{2}$ (Assef et al. 2015a).

\footnotetext{
5 http://wise2.ipac.caltech.edu/docs/release/allwise/
}

Table 1

The Sample of Hot DOGs

\begin{tabular}{lccc}
\hline \hline Source & R.A. & decl. & Redshift \\
Name & $(J 2000)$ & $(J 2000)$ & \\
\hline W0126-0529 & $01: 26: 11.96$ & $-05: 29: 09.6$ & 2.937 \\
W0134-2922 & $01: 34: 35.71$ & $-29: 22: 45.4$ & 3.047 \\
W0149+2350 & $01: 49: 46.16$ & $+23: 50: 14.6$ & 3.228 \\
W0220+0137 & $02: 20: 52.12$ & $+01: 37: 11.6$ & 3.122 \\
W0248+2705 & $02: 48: 58.81$ & $+27: 05: 29.8$ & 2.210 \\
W0410-0913 & $04: 10: 10.60$ & $-09: 13: 05.2$ & 3.592 \\
W0533-3401 & $05: 33: 58.44$ & $-34: 01: 34.5$ & 2.904 \\
W0615-5716 & $06: 15: 11.07$ & $-57: 16: 14.6$ & 3.399 \\
W0757+5113 & $07: 57: 25.07$ & $+51: 13: 19.7$ & 2.277 \\
W0859+4823 & $08: 59: 29.94$ & $+48: 23: 02.3$ & 3.245 \\
W1136+4236 & $11: 36: 34.31$ & $+42: 36: 02.6$ & 2.390 \\
W1248-2154 & $12: 48: 15.21$ & $-21: 54: 20.4$ & 3.318 \\
W1603+2745 & $16: 03: 57.39$ & $+27: 45: 53.3$ & 2.633 \\
W1814+3412 & $18: 14: 17.30$ & $+34: 12: 25.0$ & 2.452 \\
W1835+4355 & $18: 35: 33.71$ & $+43: 55: 49.1$ & 2.298 \\
W2054+0207 & $20: 54: 25.69$ & $+02: 07: 11.0$ & 2.520 \\
W2201+0226 & $22: 01: 23.39$ & $+02: 26: 21.8$ & 2.877 \\
W2210-3507 & $22: 10: 11.87$ & $-35: 07: 20.0$ & 2.814 \\
W2216+0723 & $22: 16: 19.09$ & $+07: 23: 54.5$ & 1.680 \\
W2238+2653 & $22: 38: 10.20$ & $+26: 53: 19.8$ & 2.405 \\
W2246-0526 & $22: 46: 07.57$ & $-05: 26: 35.0$ & 4.593 \\
W2305-0039 & $23: 05: 25.88$ & $-00: 39: 25.7$ & 3.106 \\
\hline
\end{tabular}

In order to investigate the detailed IR SEDs of Hot DOGs, we select a subsample of 22 objects (Table 1) from the full sample. We require that all of them have known spectroscopic redshift $z>1.5$ in the literature (Wu et al. 2012; Jones et al. 2014; Tsai et al. 2015). We also require that they have both Herschel PACS and SPIRE observations and have either SPIRE $500 \mu \mathrm{m}$ or SCUBA-2 $850 \mu \mathrm{m}$ detection, which corresponds to $>100 \mu \mathrm{m}$ at rest frame. By imposingsubmm detection, we will select those objects with 7 and even more detections between the observed $12 \mu \mathrm{m}$ and mm bands. Thanks to the submm detection, the properties of cold dust components can be well-constrained, such as IR luminosity and cold dust temperature (see Tables 3, 5), according to the IR SED decomposition. We note that we are most likely biasing our sample toward the most intense star-forming systems. We can quantify the maximal contribution of star formation in this specific class of objects and its contribution to the total IR luminosity. We are therefore assured that we will estimate meaningful upper limits on the expected maximal star formation contribution for Hot DOGs.

\subsection{Photometry}

The WISE W3 and W4 photometry for the Hot DOG sample discussed in this work is from the ALLWISE Data Release (Cutri et al. 2013). W3 and W4 flux densities and uncertainties (see Table 2) have been converted from catalog Vega magnitude by using zero points of 29.04 and $8.284 \mathrm{Jy}$, respectively (Wright et al. 2010).

We also listed the FIR photometry of our 22 Hot DOGs obtained with the Herschel Space Observatory (Pilbratt et al. 2010) in Table 2. The Herschel data (PI: P.R.M. Eisenhardt) include both PACS (Photodetector Array Camera and Spectrometer; Poglitsch et al. 2010) observations at 70 and $160 \mu \mathrm{m}$ and SPIRE (Spectral and Photometric Imaging REceiver; Griffin et al. 2010) observations at 250,350, and $500 \mu \mathrm{m}$. We retrieved 
Table 2

Photometry of Hot DOGs

\begin{tabular}{|c|c|c|c|c|c|c|c|c|c|}
\hline $\begin{array}{l}\text { Source } \\
\text { Name }\end{array}$ & $\begin{array}{c}12 \mu \mathrm{m} \\
(\mathrm{mJy})\end{array}$ & $\begin{array}{c}22 \mu \mathrm{m} \\
(\mathrm{mJy})\end{array}$ & $\begin{array}{c}70 \mu \mathrm{m} \\
(\mathrm{mJy})\end{array}$ & $\begin{array}{c}160 \mu \mathrm{m} \\
(\mathrm{mJy})\end{array}$ & $\begin{array}{c}250 \mu \mathrm{m} \\
(\mathrm{mJy})\end{array}$ & $\begin{array}{c}350 \mu \mathrm{m} \\
(\mathrm{mJy})\end{array}$ & $\begin{array}{c}500 \mu \mathrm{m} \\
(\mathrm{mJy})\end{array}$ & $\begin{array}{c}850 \mu \mathrm{m} \\
(\mathrm{mJy})\end{array}$ & $\begin{array}{c}1100 \mu \mathrm{m} \\
(\mathrm{mJy})\end{array}$ \\
\hline W0126-0529 & $1.0 \pm 0.1$ & $27.2 \pm 1.2$ & $34.8 \pm 3.5$ & $231.8 \pm 10.8$ & $204.6 \pm 5.7$ & $132.6 \pm 6.9$ & $61.7 \pm 7.0$ & $\ldots$ & $\ldots$ \\
\hline W0134-2922 & $4.6 \pm 0.2$ & $19.0 \pm 1.4$ & $24.5 \pm 4.6$ & $41.8 \pm 8.9$ & $49.1 \pm 6.1$ & $26.9 \pm 6.5$ & $36.3 \pm 7.7$ & $\ldots$ & $\ldots$ \\
\hline $\mathrm{W} 0149+2350$ & $1.8 \pm 0.1$ & $9.2 \pm 0.8$ & $29.0 \pm 4.0$ & $56.4 \pm 11.0$ & $42.9 \pm 5.4$ & $38.7 \pm 6.9$ & $33.9 \pm 10.3$ & & $2.0 \pm 0.4^{\mathrm{a}}$ \\
\hline $\mathrm{W} 0220+0137$ & $1.8 \pm 0.1$ & $12.0 \pm 0.8$ & $65.4 \pm 3.6$ & $119.0 \pm 10.8$ & $95.0 \pm 5.0$ & $77.9 \pm 7.0$ & $39.0 \pm 6.4$ & $\ldots$ & $6.2 \pm 2.0^{\mathrm{b}}$ \\
\hline W0248+2705 & $2.0 \pm 0.1$ & $11.1 \pm 1.0$ & $21.5 \pm 4.8$ & $81.6 \pm 15.1$ & $57.3 \pm 5.0$ & $47.6 \pm 6.5$ & $26.3 \pm 7.1$ & $\ldots$ & $<3.6^{\mathrm{b}}$ \\
\hline W0410-0913 & $2.5 \pm 0.1$ & $12.4 \pm 1.0$ & $36.0 \pm 4.6$ & $107.9 \pm 13.1$ & $124.4 \pm 4.7$ & $128.8 \pm 5.6$ & $99.0 \pm 6.0$ & $40.0 \pm 14.0^{\mathrm{c}}$ & $13.6 \pm 2.6^{\mathrm{b}}$ \\
\hline W0533-3401 & $3.0 \pm 0.1$ & $11.9 \pm 1.1$ & $39.3 \pm 5.9$ & $97.4 \pm 14.0$ & $107.5 \pm 4.8$ & $76.3 \pm 7.3$ & $48.9 \pm 4.5$ & $\ldots$ & $\ldots$ \\
\hline W0615-5716 & $2.2 \pm 0.1$ & $14.8 \pm 0.8$ & $56.6 \pm 2.9$ & $93.2 \pm 7.8$ & $51.4 \pm 5.2$ & $38.0 \pm 6.9$ & $28.4 \pm 6.4$ & $\ldots$ & $\ldots$ \\
\hline W0757+5113 & $1.5 \pm 0.1$ & $9.3 \pm 0.8$ & $20.1 \pm 3.5$ & $32.9 \pm 19.5$ & $44.4 \pm 5.3$ & $44.1 \pm 6.3$ & $30.7 \pm 6.6$ & & $<4.7^{\mathrm{b}}$ \\
\hline W0859+4823 & $2.2 \pm 0.1$ & $11.8 \pm 0.9$ & $37.5 \pm 3.8$ & $33.8 \pm 11.2$ & $63.6 \pm 4.9$ & $71.1 \pm 6.0$ & $51.4 \pm 6.2$ & & $6.2 \pm 1.5^{\mathrm{b}}$ \\
\hline $\mathrm{W} 1136+4236$ & $1.6 \pm 0.1$ & $7.1 \pm 0.7$ & $<13.5$ & $101.7 \pm 15.2$ & $92.3 \pm 4.6$ & $89.1 \pm 5.6$ & $58.9 \pm 5.7$ & $5.3 \pm 1.7^{\mathrm{d}}$ & $\ldots$ \\
\hline W1248-2154 & $2.6 \pm 0.1$ & $12.9 \pm 0.9$ & $54.5 \pm 4.2$ & $61.5 \pm 8.7$ & $56.6 \pm 5.1$ & $42.9 \pm 5.4$ & $20.8 \pm 5.0$ & $\ldots$ & $\ldots$ \\
\hline $\mathrm{W} 1603+2745$ & $3.2 \pm 0.1$ & $10.1 \pm 1.0$ & $13.4 \pm 3.3$ & $66.4 \pm 11.0$ & $69.0 \pm 5.0$ & $55.1 \pm 5.3$ & $35.6 \pm 6.8$ & $10.2 \pm 1.8^{\mathrm{d}}$ & $\ldots$ \\
\hline W1814+3412 & $2.0 \pm 0.1$ & $14.9 \pm 1.0$ & $39.3 \pm 5.3$ & $72.7 \pm 12.5$ & $66.5 \pm 4.7$ & $48.2 \pm 4.6$ & $31.4 \pm 6.7$ & $<3.6^{\mathrm{d}}$ & $\ldots$ \\
\hline $\mathrm{W} 1835+4355$ & $6.3 \pm 0.2$ & $27.7 \pm 1.0$ & $45.5 \pm 4.2$ & $100.5 \pm 12.5$ & $94.0 \pm 5.0$ & $80.8 \pm 5.6$ & $38.6 \pm 5.4$ & $8.0 \pm 1.5^{\mathrm{d}}$ & $\ldots$ \\
\hline W2054+0207 & $4.2 \pm 0.2$ & $11.6 \pm 1.0$ & $15.6 \pm 4.8$ & $73.7 \pm 10.4$ & $36.4 \pm 4.4$ & $35.6 \pm 4.0$ & $29.5 \pm 7.2$ & $<3.6^{\mathrm{d}}$ & $\ldots$ \\
\hline $\mathrm{W} 2201+0226$ & $4.5 \pm 0.2$ & $17.8 \pm 1.5$ & $23.6 \pm 4.4$ & $134.6 \pm 9.5$ & $156.1 \pm 5.7$ & $136.2 \pm 7.8$ & $76.0 \pm 6.1$ & $\ldots$ & $\ldots$ \\
\hline W2210-3507 & $2.1 \pm 0.1$ & $16.4 \pm 1.1$ & $55.1 \pm 3.7$ & $117.3 \pm 14.6$ & $123.9 \pm 5.9$ & $126.4 \pm 5.1$ & $101.5 \pm 6.0$ & $\ldots$ & $\ldots$ \\
\hline $\mathrm{W} 2216+0723$ & $3.2 \pm 0.2$ & $14.3 \pm 1.2$ & $59.4 \pm 3.5$ & $130.9 \pm 9.0$ & $88.3 \pm 4.9$ & $57.9 \pm 5.6$ & $<21.6$ & $5.5 \pm 1.6^{\mathrm{d}}$ & $\ldots$ \\
\hline $\mathrm{W} 2238+2653$ & $2.3 \pm 0.1$ & $17.1 \pm 1.0$ & $62.3 \pm 5.4$ & $141.7 \pm 11.9$ & $133.9 \pm 5.4$ & $94.0 \pm 5.3$ & $62.3 \pm 5.9$ & $\ldots$ & $6.0 \pm 2.2^{b}$ \\
\hline W2246-0526 & $2.3 \pm 0.2$ & $15.8 \pm 1.6$ & $29.0 \pm 4.1$ & $125.3 \pm 11.6$ & $104.0 \pm 3.9$ & $78.6 \pm 5.8$ & $52.4 \pm 5.2$ & $11.4 \pm 2.1^{\mathrm{d}}$ & $\ldots$ \\
\hline W2305-0039 & $3.2 \pm 0.2$ & $24.4 \pm 1.4$ & $23.7 \pm 2.7$ & $128.4 \pm 13.4$ & $101.8 \pm 4.9$ & $74.4 \pm 5.3$ & $58.4 \pm 6.2$ & $\ldots$ & $\ldots$ \\
\hline
\end{tabular}

Notes.

a Flux density at $1.3 \mathrm{~mm}$ obtained by the SMA (Wu et al. 2014).

${ }^{\mathrm{b}}$ Flux density or $(2 \sigma)$ upper limits at $1.1 \mathrm{~mm}$ from CSO/Bolocam (Wu et al. 2012).

${ }^{\mathrm{c}}$ Flux density at $850 \mu \mathrm{m}$ obtained by the CSO/SHARC-II (Wu et al. 2012).

${ }^{\mathrm{d}}$ Flux density or $(2 \sigma)$ upper limits at $850 \mu \mathrm{m}$ from JCMT/SCUBA-2 (Jones et al. 2014).

the Herschel data via the Herschel Science Archive (HSA). ${ }^{6}$ Both PACS and SPIRE data were reduced using the Herschel Interactive Processing Environment (HIPE v12.1.0). For PACS fluxes, we retrieved the PACS data from the HSA and reduced them with the provided PACS photometer pipeline for minimap and central point source in HIPE v12.1.0, leaving all options at their default values. After applying a mask as a combination of a central $20^{\prime \prime}$ radius mask and pixels at a signal-to-noise $>3$ on the rest of the image, highpass filtering, and MMT de-glitching were applied on the masked scans. Finally, a mosaic was created with the two reduced scans. Aperture photometry was performed with an aperture radius of $14^{\prime \prime}\left(17^{\prime \prime}\right)$ and a circle at $18^{\prime \prime}\left(36^{\prime \prime}\right)$ and $24^{\prime \prime}\left(48^{\prime \prime}\right)$ radius in the blue (red) channel to estimate the local background level. Uncertainties were calculated by placing the aperture in the image $\left(>48^{\prime \prime}\right)$ around the source. The final uncertainties were taken as the median absolute deviation of these apertures. For SPIRE fluxes, we retrieved the pre-reduced data from the archive and applied the script to execute point source photometry directly on the level 2 maps (provided in the HIPE scripts). The SUSSextractor task was used and their associated uncertainties were derived with aperture photometry, assuming $22^{\prime \prime}, 30, "$ and $42^{\prime \prime}$ radii for the 250,350 , and $500 \mu \mathrm{m}$ channels, respectively. The uncertainties were calculated as the quadratic sum of the background fluctuation (assuming an annulus with inner and outer circles of $60^{\prime \prime}$ and $90^{\prime \prime}$ respectively) and the photon noise of the source in the previously calculated aperture.

Seven objects in our Hot DOG sample had JCMT SCUBA-2 $850 \mu \mathrm{m}$ submm observations (Jones et al. 2014). W0410-0913

\footnotetext{
http://www.cosmos.esa.int/web/herschel/science-archive
}

had been detected at $850 \mu \mathrm{m}$ with CSO SHARC-II in Wu et al. (2012). Six Hot DOGs had CSO Bolocam observations at $1.1 \mathrm{~mm}$ (Wu et al. 2012). W0149+2350 had $1.3 \mathrm{~mm}$ detection obtained by the SMA (Wu et al. 2014). All the available submm and mm photometry has also been listed in Table 2 .

\section{IR SED DECOMPOSITION}

The IR emission of Hot DOGs could come from the hotter AGN-heated dust emission and/or colder young stellarpopulation-heated dust emission. To understand the principal physical processes responsible for the luminous IR emission of these galaxies, we need to determine the relative contributions of the two components. Then, in order to decompose the IR SEDs of Hot DOGs for the two components, we need a model for each of them. For the AGN-heated dust emission, which contributes mainly to the mid-IR emission, we have employed the CLUMPY torus model by Nenkova et al. (2002, 2008a, $2008 \mathrm{~b}){ }^{7}$ For the young stellar-population-heated dust emission, which contributes mainly to the FIR emission, we have employed a simple modified blackbody (MBB, or graybody) model.

We use an updated version of the Bayesian SED fitting code BayeSED (Han \& Han 2012, 2014) to decompose the IR SED of Hot DOGs using a new version of the CLUMPY torus model and a simple graybody model to represent the contribution of dust emission heated by young stellar population. A detailed description of BayeSED can be found in Appendix A. 
We use the newly calculated CLUMPY model database. ${ }^{8}$ There are 1,247,400 models in the database, with 119 wavelengths for each SED. The torus-only model SEDs, which are stored in flux $x_{\text {tor }}$, are used in this paper. Instead of the ANN method as employed in Han \& Han (2012), we use the KNN method to interpolate these model SEDs. As shown in Han \& Han (2014), the KNN method results in a better interpolation of SEDs, though it leads to a larger data file. The size of the original database, which is provided as an HDF5 file, is 1.2 GB. With the machine learning methods employed in BayeSED, it is reduced to only $180 \mathrm{MB}$ without noticeable loss of information (we have ignored the principal components with variation less than $0.01 \%$ of the total, and have used the left 21 principal components). The CLUMPY torus model has 6 parameters: the number of clouds along a radial equatorial path $N_{0}$, the ratio of the outer to the inner radii of the toroidal distribution $Y=R_{\mathrm{o}} / R_{\mathrm{d}}$, the viewing angle measured from the torus polar axis $i$, the index $q$ of the radial density profile $r^{-q}$, the width parameter characterizing the angular distribution $\sigma$, and the effective optical depth of clumps $\tau_{\mathrm{V}}$. The priors for the 6 parameters are assumed to be uniform distributions truncated to the following intervals: $N_{0}=[1,15], \quad Y=[5,100]$, $i=[0,90], q=[0,3], \sigma=[15,70], \tau_{\mathrm{V}}=[10,300]$.

Two more quantities have been defined by Nenkova et al. (2008a) to describe the dust-covering of AGNs. One is the probability that a photon emitted by the AGN in direction of the given inclination angle of the torus with respect to the line of sight will escape the obscuring structure, or in other words, the probability that the object can be observed as a Type 1 AGN $\left(P_{\text {type } 1}\right)$. The other is the geometrical dust-covering factor (CF) of the torus, $f_{2}$, which is also the average of the fraction of the AGN radiation absorbed by obscuring clouds. These two quantities can be set by the six free parameters of the CLUMPY model. Assuming optically thick clouds, $P_{\text {type } 1}$ can be approximately written as a function of the inclination angle, $i$ :

$$
P_{\text {type1 }}=e^{-N_{0} e^{-\frac{\theta^{2}}{\sigma^{2}}}}
$$

where $\theta=\pi / 2-i$. The geometrical dust $\mathrm{CF}, f_{2}$, can be derived by integrating $P_{\text {type } 1}$ and subtracting from 1 (Nenkova et al. 2008a; Mor et al. 2009):

$$
f_{2}=1-\int_{0}^{\pi / 2} P_{\text {typel }} \cos (\theta) d \theta
$$

The graybody model is defined as:

$$
S_{\lambda} \propto\left(1-e^{-\left(\frac{\lambda_{0}}{\lambda}\right)^{\beta}}\right) B_{\lambda}\left(T_{\text {dust }}\right)
$$

where $B_{\lambda}$ is the Planck blackbody spectrum, $T_{\text {dust }}$ is dust temperature, and we use the typical value of $\lambda_{0}=125 \mu \mathrm{m}$. We adopt $\beta=1.6$, which is the value typically used for highredshift QSOs (Beelen et al. 2006; Wang et al. 2008, 2011). So, the dust temperature $T_{\text {dust }}$ is the only free parameter with a uniform prior truncated to the interval of $\log \left(T_{\text {dust }} / K\right)=[1,2]$.

\footnotetext{
8 http://www.pa.uky.edu/clumpy/models/

clumpy_models_201410_tvavg.hdf5 /
}

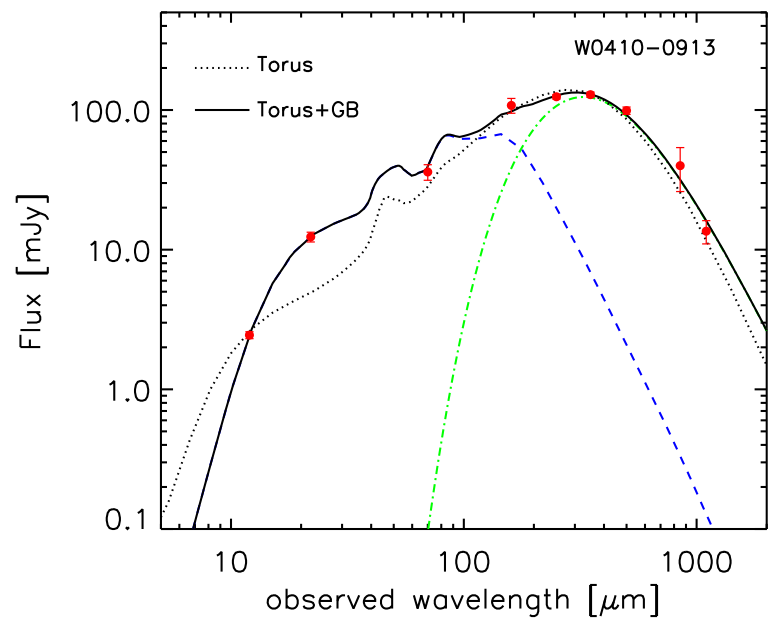

Figure 1. Observed SED of a Hot DOG, W0410-0913 (filled circles), together with model fitting. The dotted line shows a torus-only model fit and the solid line represents the combined Torus+GB model. The dashed and dot-dashed lines represent the components of the torus and graybody in the Torus+GB model, respectively. As listed in Table 4, its natural logarithm of the Bayes factor (Jeffreys \& Oxford 1961) $\ln \left(\frac{\text { evTORUS }+\mathrm{GB}}{\text { evtorus }}\right)=37.37$ presents strong evidence in favor of the Torus + GB model.

\section{RESULTS}

\subsection{Model Comparison}

Previous works found that the IR SEDs of Hot DOGs are very similar, showing a steep spectrum at $1-10 \mu \mathrm{m}$ that is due to the selection criteria of Hot DOGs. Compared to various galaxy SED templates in Polletta et al. (2007), such as Arp 220 (starburst galaxy), Mrk 231 (heavily obscured AGN and starburst composite), QSO1 and QSO2 (optically selected Type 1 and Type 2 QSOs), the mid-IR to submm SEDs of Hot DOGs appear to be flatter (Wu et al. 2012; Jones et al. 2014; Tsai et al. 2015). The obvious difference between the SEDs of Hot DOGs and the compared galaxy SED templates prompts us to fit the IR SEDs of Hot DOGs with other models.

At first, we use a torus-only model of Nenkova et al. (2002, 2008a, 2008b), as presented in the CLUMPY library (noted as hereafter Torus) to fit the IR SEDs of all Hot DOGs. Then we use a combined model, the torus plus a graybody (Torus $+\mathrm{GB}$ ) component, to do the SED decomposition. The presence of significant star formation activity in Hot DOGs has also been suggested by Frey et al. (2016). They found that the sum of the VLBI component flux densities is always smaller than the total flux density, indicating that star formation activity in the host galaxy should be responsible for the missing flux density. In Figure 1, we show an example of IR SED fitting results with Torus (dotted line) and Torus+GB model (solid line), respectively. In the case of W0410-0913 (Figure 1), the Torus $+\mathrm{GB}$ model seems to provide a better fitting to the observations than Torus model. However, the Torus+GB model also introduces one more free parameter than the Torus model.

In order to compare different models quantitatively, we derive their Bayesian evidence, which represents a practical implementation of the Occam's razor principle. In our case, the Torus+GB model with more parameters will have lower Bayesian evidence unless it provides a significantly better fitting than the Torus model. In Table 4, we present the natural logarithm $\ln \left(\mathrm{ev}_{\text {TORUS }}\right)$ and $\ln \left(\mathrm{ev}_{\mathrm{TORUS}+\mathrm{GB}}\right)$ of the Bayesian 
Table 3

Luminosities of Hot DOGs

\begin{tabular}{|c|c|c|c|}
\hline Source & $\begin{array}{c}\log L_{\mathrm{IR}}^{t} \\
\left(L_{\odot}\right)\end{array}$ & $\begin{array}{c}\log L_{\mathrm{IR}}^{\mathrm{cd}} \\
\left(L_{\odot}\right)\end{array}$ & $\begin{array}{c}\log L_{\mathrm{IR}}^{\mathrm{tot}} \\
\left(L_{\odot}\right)\end{array}$ \\
\hline W0126-0529 & $13.98_{-0.01}^{+0.01}$ & $13.91_{-0.01}^{+0.01}$ & $14.25_{-0.01}^{+0.01}$ \\
\hline W0134-2922 & $14.02_{-0.02}^{+0.01}$ & $13.20_{-0.04}^{+0.04}$ & $14.08_{-0.02}^{+0.02}$ \\
\hline W0149+2350 & $13.89_{-0.02}^{+0.02}$ & $13.23_{-0.05}^{+0.05}$ & $13.98_{-0.03}^{+0.02}$ \\
\hline $\mathrm{W} 0220+0137$ & $14.08_{-0.02}^{+0.02}$ & $13.52_{-0.07}^{+0.06}$ & $14.19_{-0.03}^{+0.03}$ \\
\hline $\mathrm{W} 0248+2705$ & $13.45_{-0.02}^{+0.02}$ & $13.06_{-0.05}^{+0.05}$ & $13.60_{-0.03}^{+0.03}$ \\
\hline W0410-0913 & $14.20_{-0.02}^{+0.02}$ & $13.70_{-0.02}^{+0.03}$ & $14.31_{-0.02}^{+0.02}$ \\
\hline W0533-3401 & $13.88_{-0.02}^{+0.02}$ & $13.54_{-0.03}^{+0.03}$ & $14.05_{-0.02}^{+0.02}$ \\
\hline W0615-5716 & $14.24_{-0.01}^{+0.01}$ & $13.09_{-0.14}^{+0.13}$ & $14.27_{-0.02}^{+0.02}$ \\
\hline W0757+5113 & $13.42_{-0.02}^{+0.02}$ & $12.79_{-0.03}^{+0.03}$ & $13.52_{-0.02}^{+0.02}$ \\
\hline W0859+4823 & $14.00_{-0.01}^{+0.01}$ & $13.32_{-0.02}^{+0.01}$ & $14.08_{-0.01}^{+0.01}$ \\
\hline $\mathrm{W} 1136+4236$ & $13.61_{-0.08}^{+0.08}$ & $13.23_{-0.04}^{+0.04}$ & $13.76_{-0.07}^{+0.07}$ \\
\hline W1248-2154 & $14.13_{-0.01}^{+0.01}$ & $13.32_{-0.04}^{+0.05}$ & $14.19_{-0.02}^{+0.02}$ \\
\hline $\mathrm{W} 1603+2745$ & $13.61_{-0.02}^{+0.02}$ & $13.24_{-0.03}^{+0.03}$ & $13.77_{-0.02}^{+0.02}$ \\
\hline $\mathrm{W} 1814+3412$ & $13.72_{-0.02}^{+0.02}$ & $13.18_{-0.04}^{+0.04}$ & $13.83_{-0.02}^{+0.02}$ \\
\hline $\mathrm{W} 1835+4355$ & $13.89_{-0.01}^{+0.01}$ & $13.29_{-0.03}^{+0.02}$ & $13.99_{-0.01}^{+0.01}$ \\
\hline W2054+0207 & $13.67_{-0.02}^{+0.02}$ & $13.09_{-0.05}^{+0.05}$ & $13.77_{-0.03}^{+0.02}$ \\
\hline $\mathrm{W} 2201+0226$ & $13.92_{-0.02}^{+0.01}$ & $13.71_{-0.01}^{+0.01}$ & $14.13_{-0.01}^{+0.01}$ \\
\hline W2210-3507 & $13.97_{-0.01}^{+0.01}$ & $13.47_{-0.01}^{+0.01}$ & $14.09_{-0.01}^{+0.01}$ \\
\hline $\mathrm{W} 2216+0723$ & $13.37_{-0.03}^{+0.03}$ & $13.15_{-0.04}^{+0.04}$ & $13.58_{-0.04}^{+0.03}$ \\
\hline $\mathrm{W} 2238+2653$ & $13.83_{-0.01}^{+0.01}$ & $13.47_{-0.02}^{+0.02}$ & $13.99_{-0.02}^{+0.02}$ \\
\hline W2246-0526 & $14.46_{-0.02}^{+0.01}$ & $13.73_{-0.05}^{+0.04}$ & $14.53_{-0.02}^{+0.02}$ \\
\hline W2305-0039 & $14.03_{-0.01}^{+0.01}$ & $13.61_{-0.02}^{+0.02}$ & $14.17_{-0.01}^{+0.01}$ \\
\hline
\end{tabular}

evidence for the Torus and Torus+GB models. We also present the natural logarithm of the Bayes factor $\ln \left(\frac{\mathrm{ev}_{\mathrm{TORUS}+\mathrm{GB}}}{\mathrm{ev}_{\mathrm{TORUS}}}\right)$ in Table 4. We find that the Torus+GB model has higher Bayesian evidence than the Torus model for all Hot DOGs. We also find that $\ln \left(\frac{\mathrm{ev}_{\text {ToRUS }+\mathrm{GB}}}{\mathrm{ev}_{\mathrm{TORUS}}}\right)>10$ (corresponding to odds of $>20000$ : 1), which presents strong evidence in favor of the Torus + GB model according to the empirically calibrated Jeffreys's scale (Jeffreys \& Oxford 1961; Trotta 2008). Thereafter, we use the results of the SED fitting with the Torus+GB model.

\subsection{Model Parameters}

Our Bayesian analysis of SEDs has the advantage of providing a detailed posterior distribution for the free and derived parameters. From these probability distributions, we can derive the best expectations and uncertainties of all the parameters. From the detailed posterior probability distributions of all parameters, it is easy to find out if a parameter is well-constrained or not. Figure 2 shows the one-dimensional and two-dimensional marginalized posterior probability distributions of the seven free parameters, including six free parameters $\left(Y, i, q, \sigma, N_{0}, \tau_{V}\right)$ for the torus model and one free parameter $\left(T_{\text {dust }}\right)$ for the graybody model, and for one Hot DOG W0410-0913 as an example. We can see that the graybody temperature $T_{\text {dust }}$ is tightly constrained: $T_{\text {dust }}$ is constrained to a narrow range, around $\sim 63 \mathrm{~K}$. However, some parameters are loosely constrained: for example, $i$, the inclination angle of the torus with respect to the line of sight, and $Y$, the ratio between the radius of the torus and the dust sublimation radius, are rather weakly constrained.

Figure 3 shows the one-dimensional and two-dimensional marginalized posterior probability distributions of our derived quantities: the geometrical $\mathrm{CF}$ of the torus $\left(f_{2}\right)$, the probability
Table 4

The Bayesian Evidence of the "TORUS" and "TORUS+GB" Models

\begin{tabular}{|c|c|c|c|}
\hline Source & $\ln \left(\mathrm{eV}_{\text {TORUS }}\right)$ & $\ln \left(\mathrm{ev}_{\mathrm{TORUS}}+\mathrm{GB}\right)$ & $\ln \left(\frac{\text { evTORUS }+\mathrm{GB}}{\text { evTORUS }}\right)$ \\
\hline W0126-0529 & $-158.38 \pm 0.18$ & $-71.88 \pm 0.23$ & $86.50 \pm 0.41$ \\
\hline W0134-2922 & $-62.42 \pm 0.13$ & $-15.26 \pm 0.14$ & $47.16 \pm 0.28$ \\
\hline $\mathrm{W} 0149+2350$ & $-34.56 \pm 0.14$ & $-9.15 \pm 0.12$ & $25.41 \pm 0.26$ \\
\hline $\mathrm{W} 0220+0137$ & $-49.31 \pm 0.17$ & $-9.25 \pm 0.13$ & $40.06 \pm 0.30$ \\
\hline W0248+2705 & $-34.11 \pm 0.13$ & $-9.43 \pm 0.12$ & $24.68 \pm 0.24$ \\
\hline W0410-0913 & $-49.89 \pm 0.17$ & $-12.51 \pm 0.14$ & $37.37 \pm 0.31$ \\
\hline W0533-3401 & $-28.80 \pm 0.14$ & $-7.65 \pm 0.12$ & $21.15 \pm 0.26$ \\
\hline W0615-5716 & $-28.50 \pm 0.16$ & $-11.74 \pm 0.15$ & $16.76 \pm 0.31$ \\
\hline $\mathrm{W} 0757+5113$ & $-42.14 \pm 0.12$ & $-7.73 \pm 0.12$ & $34.41 \pm 0.24$ \\
\hline W0859+4823 & $-68.60 \pm 0.16$ & $-9.93 \pm 0.13$ & $58.68 \pm 0.29$ \\
\hline W1136+4236 & $-39.94 \pm 0.15$ & $-19.71 \pm 0.10$ & $20.23 \pm 0.25$ \\
\hline W1248-2154 & $-34.39 \pm 0.17$ & $-7.84 \pm 0.13$ & $26.55 \pm 0.29$ \\
\hline $\mathrm{W} 1603+2745$ & $-59.20 \pm 0.18$ & $-10.33 \pm 0.13$ & $48.87 \pm 0.30$ \\
\hline $\mathrm{W} 1814+3412$ & $-62.11 \pm 0.15$ & $-9.49 \pm 0.14$ & $52.62 \pm 0.29$ \\
\hline $\mathrm{W} 1835+4355$ & $-191.90 \pm 0.19$ & $-13.28 \pm 0.15$ & $178.62 \pm 0.34$ \\
\hline W2054+0207 & $-53.24 \pm 0.14$ & $-15.48 \pm 0.11$ & $37.77 \pm 0.25$ \\
\hline $\mathrm{W} 2201+0226$ & $-60.11 \pm 0.18$ & $-14.92 \pm 0.15$ & $45.19 \pm 0.33$ \\
\hline W2210-3507 & $-112.54 \pm 0.16$ & $-18.67 \pm 0.14$ & $93.87 \pm 0.31$ \\
\hline $\mathrm{W} 2216+0723$ & $-25.41 \pm 0.14$ & $-7.15 \pm 0.11$ & $18.25 \pm 0.26$ \\
\hline $\mathrm{W} 2238+2653$ & $-90.39 \pm 0.16$ & $-11.86 \pm 0.14$ & $78.52 \pm 0.30$ \\
\hline W2246-0526 & $-32.61 \pm 0.14$ & $-22.29 \pm 0.16$ & $10.32 \pm 0.30$ \\
\hline W2305-0039 & $-128.15 \pm 0.17$ & $-43.15 \pm 0.20$ & $85.01 \pm 0.36$ \\
\hline
\end{tabular}

that light from the central source can escape the obscuring structure without interacting with the clouds and therefore the object, can be observed as a Type 1 AGN $\left(P_{\text {type } 1}\right)$, and $1-1000 \mu \mathrm{m}$ IR luminosities of the torus $\left(L_{\mathrm{IR}}^{t}\right)$ and cold dust $\left(L_{\mathrm{IR}}^{\mathrm{cd}}\right)$ components, for W0410-0913 as an example. Both $L_{\mathrm{IR}}^{t}$ and $L_{\mathrm{IR}}^{\mathrm{cd}}$ are well-constrained to a narrow range. And $L_{\mathrm{IR}}^{t}$ and $L_{\mathrm{IR}}^{\mathrm{cd}}$ are strongly anti-correlated. The nearly linear anticorrelation between $L_{\mathrm{IR}}^{t}$ and $L_{\mathrm{IR}}^{\text {cd }}$ indicates that $L_{\mathrm{IR}}^{\text {tot }}$, the sum of $L_{\mathrm{IR}}^{t}$ and $L_{\mathrm{IR}}^{\mathrm{cd}}$, is tightly constrained by the observed SEDs.

In order to give a good estimate for all parameters and their spreads, we use the median and percentile statistics. The lower and upper quartiles are the values below which $16 \%$ and $84 \%$ of points fall, respectively. We list the median values and the $16 \%$ and $84 \%$ quartiles of seven free parameters $\left(Y, i, q, \sigma, N_{0}, \tau_{V}, T_{\text {dust }}\right)$ and two derived quantities $\left(f_{2}, P_{\text {type } 1}\right)$ in Table 5. The other two derived quantities $\left(L_{\mathrm{IR}}^{t}\right.$ and $\left.L_{\mathrm{IR}}^{\mathrm{cd}}\right)$ are separately listed in Table 3 (see also Section 4.3).

\subsection{Luminosity Estimates}

We derive the IR luminosities of Hot DOGs based on the best-fitting results employing the Torus + GB model. Our IR SED decomposition approach enables us to derive the contributions of both torus and cold dust components to the total IR energy output. In Table 3, we listed the torus $\left(L_{\mathrm{IR}}^{\mathrm{t}}\right)$, cold dust $\left(L_{\mathrm{IR}}^{\mathrm{cd}}\right)$, and total $\left(L_{\mathrm{IR}}^{\text {tot }}\right)$ IR luminosities within the $1-1000 \mu \mathrm{m}$ range. Of 22 Hot DOGs, 12 have $L_{\mathrm{IR}}^{\text {tot }}>10^{14} L_{\odot}$, which are broadly consistent with the conservative estimates of IR luminosities in Tsai et al. (2015). Following Tsai et al. (2015), they are "extremely luminous infrared galaxies" (ELIRGs). The remaining 10 Hot DOGs have $L_{\mathrm{IR}}^{\text {tot }}>10^{13.5} L_{\odot}$, which are hyperluminous infrared galaxies (HyLIRGs). Both the distributions of $L_{\mathrm{IR}}^{t}$ and $L_{\mathrm{IR}}^{\mathrm{cd}}$ span one order of magnitude with $L_{\mathrm{IR}}^{t} \sim 10^{13.4-14.5} L_{\odot}$ and $L_{\mathrm{IR}}^{\mathrm{cd}} \sim 10^{12.8-13.9} L_{\odot}$, and peak at $10^{13.9} L_{\odot}$ and $10^{13.3} L_{\odot}$, respectively (see Figure 4). The torus IR luminosities of Hot DOGs are on average three times higher than those of cold dust. The fraction of the cold dust component to the total IR 


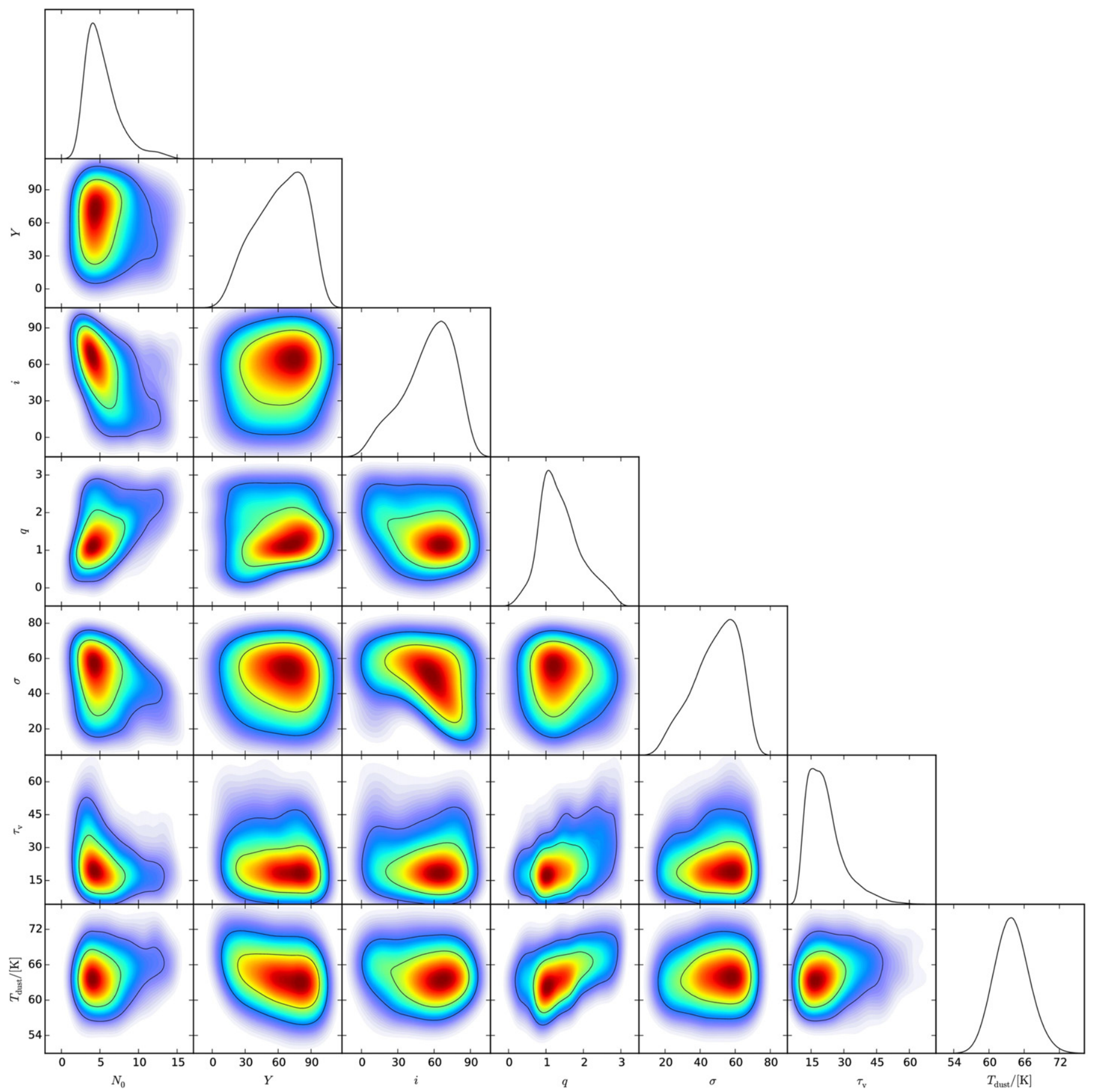

Figure 2. One-dimensional and two-dimensional marginalized posterior probability distributions of the seven free parameters, including six free parameters $\left(Y, i, q, \sigma, N_{0}, \tau_{V}\right)$ for the torus model and one free parameter $\left(T_{\text {dust }}\right)$ for the graybody model, for the Hot DOG, W0410-0913. The color-coding represents the confidence levels. Both one-dimensional and two-dimensional marginalized posterior probability distributions have been normalized to unit area.

luminosity $\left(f_{\text {cd }}\right.$, see the right panel in Figure 4$)$ ranges from 0.05 to about 0.5 , with a median value of 0.24 . This result confirms the previous argument that the IR energy output of Hot DOGs is dominated by hot dust emission in AGN tori.

We note that the relative contribution of the cold dust component is dependent on the choice of torus model. For instance, Siebenmorgen et al. (2015) presented a self-consistent AGN torus model (thereafter S15 model) ${ }^{9}$ with a different

\footnotetext{
9 www.eso.org// rsiebenm/agn_models/
}

chemical dust composition and grain geometries, predicting that the AGN torus would have stronger FIR/submm emission than the CLUMPY torus model. In this case, the contribution of the cold dust component will be lower than what we have derived. In order to test the effect of different models on the derived cold dust contribution, we replace the CLUMPY torus model with the S15 model and do the fitting again. We find that the Torus+GB model always has the highest Bayesian evidence among the sole $\mathrm{S} 15, \mathrm{~S} 15+\mathrm{GB}$, and Torus $+\mathrm{GB}$ models. As expected, the median value of $f_{\mathrm{cd}}$ derived from the 


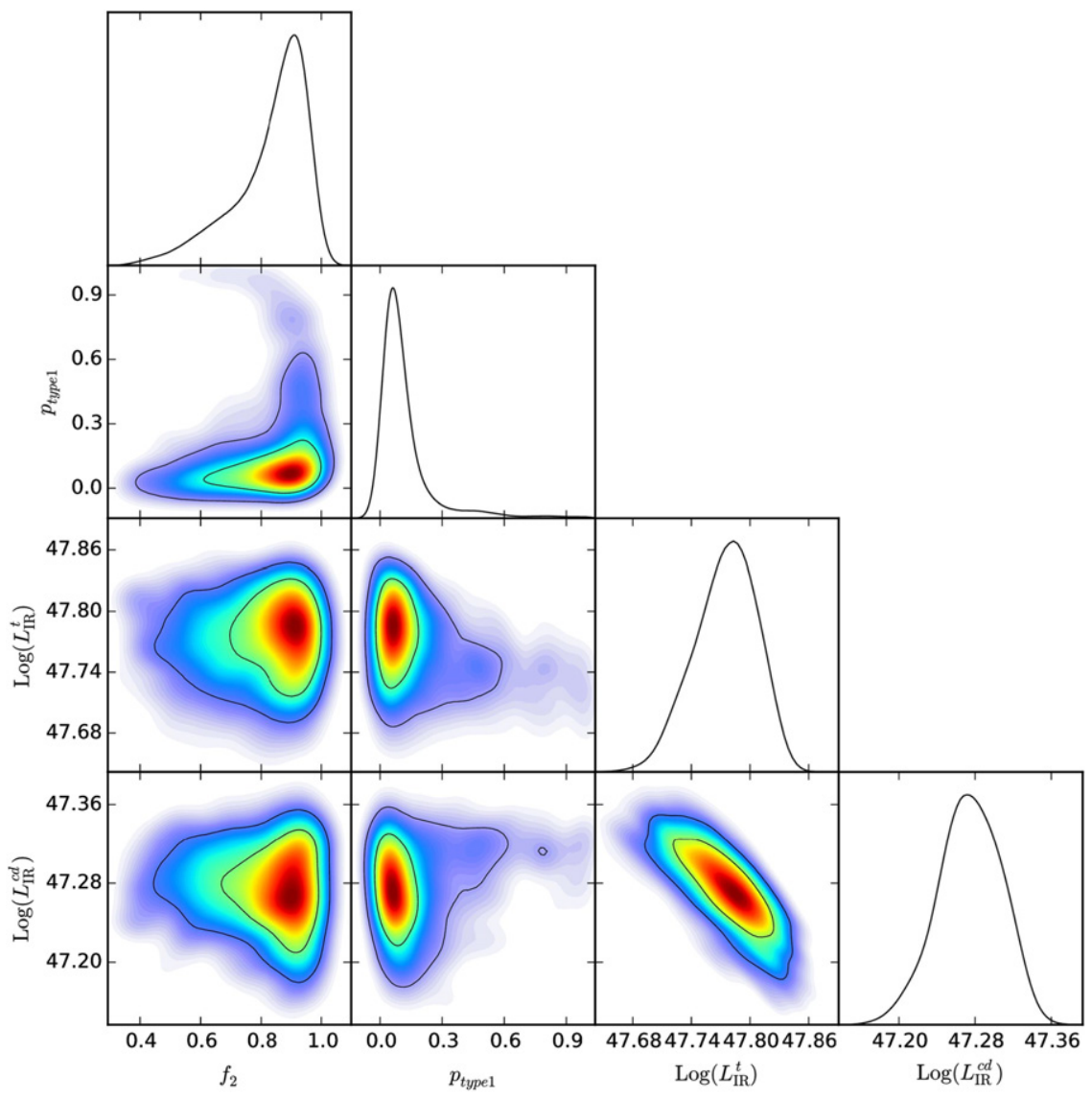

Figure 3. One-dimensional and two-dimensional marginalized posterior probability distributions of four derived quantities: the geometrical covering factor of the torus $\left(f_{2}\right)$, the probability that light from the central source can escape the obscuring structure without interacting with the clouds and therefore the object, can be observed as a Type 1 AGN $\left(P_{\text {type } 1}\right)$, and 1-1000 $\mu \mathrm{m}$ IR luminosities of the torus $\left(L_{\mathrm{IR}}^{t}\right)$ and cold dust $\left(L_{\mathrm{IR}}^{\mathrm{cd}}\right)$ components, for W0410-0913 as an example. The colorcoding represents the confidence levels. Both one-dimensional and two-dimensional marginalized posterior probability distributions have been normalized to unit area.

Table 5

The Model Parameters and Derived Quantities with the Best-fitting TORUS+GB Model

\begin{tabular}{|c|c|c|c|c|c|c|c|c|c|}
\hline Source & $N_{0}$ & $Y$ & $i$ & $q$ & $\sigma$ & $\tau_{v}$ & $f_{2}$ & $P_{\text {Type1 }}$ & $T_{\text {dust }}$ \\
\hline W0126-0529 & $13.65_{-0.16}^{+0.50}$ & $5.76_{-0.37}^{+0.59}$ & $85.67_{-0.76}^{+2.02}$ & $2.88_{-0.06}^{+0.05}$ & $67.51_{-0.50}^{+0.28}$ & $11.23_{-0.60}^{+0.75}$ & $1.00_{-0.01}^{+0.01}$ & $0.01_{-0.01}^{+0.01}$ & $88.27_{-1.25}^{+1.23}$ \\
\hline W0134-2922 & $5.16_{-0.87}^{+1.09}$ & $48.51_{-22.29}^{+23.80}$ & $64.01_{-20.53}^{+13.10}$ & $2.86_{-0.07}^{+0.07}$ & $52.80_{-9.16}^{+8.16}$ & $17.34_{-3.77}^{+5.29}$ & $0.89_{-0.10}^{+0.05}$ & $0.03_{-0.02}^{+0.14}$ & $74.94_{-5.36}^{+5.67}$ \\
\hline W0149+2350 & $6.62_{-1.78}^{+3.02}$ & $56.06_{-22.60}^{+20.51}$ & $48.02_{-25.10}^{+22.57}$ & $2.38_{-0.40}^{+0.31}$ & $49.67_{-9.28}^{+8.20}$ & $26.20_{-7.65}^{+10.89}$ & $0.91_{-0.11}^{+0.05}$ & $0.05_{-0.04}^{+0.29}$ & $75.44_{-6.17}^{+6.39}$ \\
\hline W0248+2705 & $8.41_{-2.28}^{+2.46}$ & $48.88_{-26.83}^{+25.31}$ & $42.23_{-21.42}^{+22.75}$ & $2.75_{-0.27}^{+0.11}$ & $53.32_{-7.70}^{+7.23}$ & $37.38_{-13.04}^{+22.25}$ & $0.93_{-0.10}^{+0.05}$ & $0.03_{-0.03}^{+0.36}$ & $66.74_{-5.27}^{+5.31}$ \\
\hline W0410-0913 & $5.06_{-1.18}^{+1.60}$ & $63.33_{-19.40}^{+16.46}$ & $54.89_{-20.39}^{+15.69}$ & $1.32_{-0.34}^{+0.41}$ & $50.44_{-10.47}^{+8.68}$ & $19.61_{-4.67}^{+6.20}$ & $0.89_{-0.14}^{+0.06}$ & $0.07_{-0.04}^{+0.12}$ & $63.81_{-2.12}^{+2.23}$ \\
\hline W0757+5113 & $8.92_{-2.17}^{+2.66}$ & $44.57_{-25.02}^{+26.46}$ & $53.49_{-24.07}^{+17.43}$ & $2.68_{-0.23}^{+0.16}$ & $54.12_{-8.21}^{+6.67}$ & $35.19_{-10.07}^{+14.24}$ & $0.96_{-0.06}^{+0.03}$ & $0.01_{-0.01}^{+0.16}$ & $45.43_{-3.15}^{+3.80}$ \\
\hline W0859+4823 & $7.18_{-2.02}^{+2.42}$ & $40.70_{-19.22}^{+27.54}$ & $46.42_{-22.76}^{+22.35}$ & $2.53_{-0.27}^{+0.24}$ & $51.05_{-8.26}^{+7.56}$ & $25.38_{-6.80}^{+9.17}$ & $0.93_{-0.09}^{+0.04}$ & $0.05_{-0.04}^{+0.29}$ & $59.11_{-1.98}^{+1.87}$ \\
\hline W1136+4236 & $8.68_{-2.85}^{+2.88}$ & $44.54_{-19.17}^{+27.21}$ & $27.46_{-15.22}^{+28.70}$ & $2.27_{-0.42}^{+0.35}$ & $41.54_{-8.22}^{+8.67}$ & $139.25_{-60.30}^{+63.22}$ & $0.88_{-0.12}^{+0.06}$ & $0.27_{-0.25}^{+0.67}$ & $59.84_{-3.00}^{+2.65}$ \\
\hline W1248-2154 & $6.90_{-2.04}^{+3.42}$ & $41.06_{-19.61}^{+25.37}$ & $33.40_{-18.73}^{+25.49}$ & $2.48_{-0.40}^{+0.31}$ & $46.53_{-6.75}^{+8.75}$ & $40.77_{-11.03}^{+12.56}$ & $0.90_{-0.09}^{+0.03}$ & $0.18_{-0.15}^{+0.51}$ & $81.60_{-5.48}^{+6.24}$ \\
\hline$W 1603+2745$ & $4.55_{-1.59}^{+2.45}$ & $50.87_{-23.17}^{+22.36}$ & $49.41_{-24.03}^{+19.23}$ & $2.78_{-0.25}^{+0.11}$ & $43.09_{-12.08}^{+12.13}$ & $26.13_{-8.09}^{+17.67}$ & $0.75_{-0.18}^{+0.13}$ & $0.29_{-0.25}^{+0.63}$ & $66.39_{-2.89}^{+2.90}$ \\
\hline W1814+3412 & $10.07_{-2.41}^{+2.34}$ & $40.11_{-19.79}^{+27.10}$ & $49.91_{-22.25}^{+17.99}$ & $2.72_{-0.25}^{+0.15}$ & $57.30_{-6.11}^{+5.30}$ & $29.62_{-8.25}^{+11.45}$ & $0.97_{-0.04}^{+0.02}$ & $0.00_{-0.00}^{+0.05}$ & $69.97_{-4.22}^{+4.52}$ \\
\hline W2210-3507 & $9.25_{-1.84}^{+2.31}$ & $57.34_{-24.04}^{+20.08}$ & $58.46_{-23.46}^{+16.24}$ & $2.55_{-0.26}^{+0.22}$ & $55.12_{-7.52}^{+6.65}$ & $25.45_{-6.30}^{+9.26}$ & $0.96_{-0.06}^{+0.02}$ & $0.00_{-0.00}^{+0.04}$ & $52.60_{-1.34}^{+1.51}$ \\
\hline W2216+0723 & $8.98_{-2.74}^{+2.87}$ & $49.49_{-23.74}^{+23.23}$ & $20.09_{-10.88}^{+20.56}$ & $2.49_{-0.22}^{+0.23}$ & $41.21_{-8.55}^{+8.49}$ & $169.48_{-53.83}^{+51.39}$ & $0.88_{-0.13}^{+0.05}$ & $0.39_{-0.36}^{+0.58}$ & $73.22_{-3.38}^{+3.62}$ \\
\hline $\mathrm{W} 2238+2653$ & $8.25_{-1.57}^{+2.43}$ & $45.46_{-22.87}^{+22.67}$ & $43.49_{-20.41}^{+21.06}$ & $2.66_{-0.26}^{+0.18}$ & $56.55_{-6.41}^{+5.63}$ & $49.45_{-11.50}^{+16.13}$ & $0.96_{-0.05}^{+0.02}$ & $0.01_{-0.01}^{+0.13}$ & $70.06_{-2.59}^{+2.27}$ \\
\hline W2246-0526 & $6.86_{-1.12}^{+1.45}$ & $75.70_{-13.10}^{+12.99}$ & $16.97_{-7.65}^{+11.05}$ & $0.95_{-0.16}^{+0.13}$ & $49.89_{-8.17}^{+7.93}$ & $14.06_{-1.98}^{+3.18}$ & $0.92_{-0.11}^{+0.04}$ & $0.40_{-0.16}^{+0.29}$ & $94.62_{-3.96}^{+2.79}$ \\
\hline W2305-0039 & $6.68_{-0.47}^{+0.63}$ & $6.48_{-0.77}^{+1.54}$ & $79.01_{-7.51}^{+5.89}$ & $2.89_{-0.06}^{+0.05}$ & $60.65_{-2.83}^{+4.06}$ & $12.44_{-1.28}^{+1.75}$ & $0.96_{-0.01}^{+0.01}$ & $0.00_{-0.00}^{+0.00}$ & $81.23_{-2.52}^{+2.49}$ \\
\hline
\end{tabular}



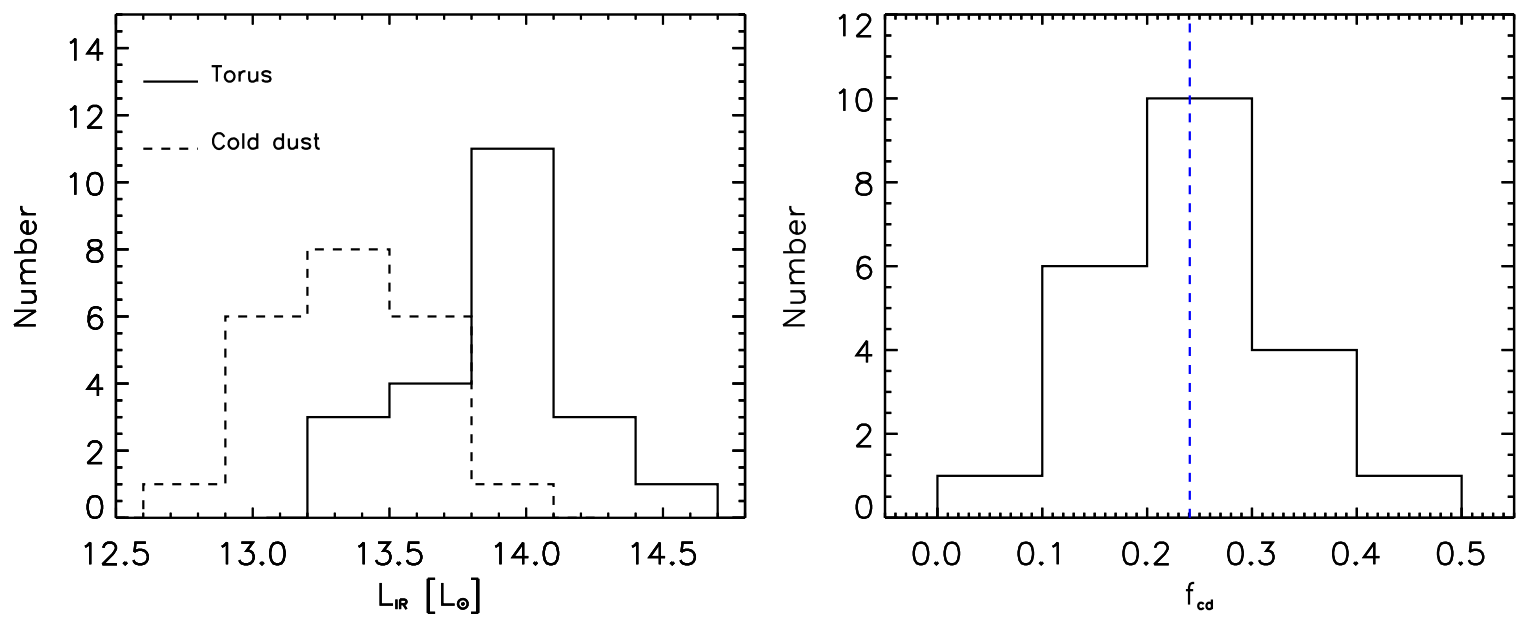

Figure 4. Left: IR luminosity distributions of torus (solid line) and cold dust components (dashed line). Right: distribution of the fraction of cold dust component to the total IR luminosity $\left(f_{\mathrm{cd}}=\frac{L_{\mathrm{IR}}^{\text {cd }}}{L_{\mathrm{IR}}^{\text {cd }}+L_{\mathrm{IR}}^{t}}\right)$. The dashed line marks the median value of $f_{\mathrm{cd}}$ at 0.24 .

S15+GB model is much lower $(\sim 0.1)$. Thus we adopt the results of the Torus $+\mathrm{GB}$ model and treat our estimation of $f_{\mathrm{cd}}$ with the Torus+GB model as the maximum possible value.

\subsection{Median SED of Hot DOGs}

In Figure 5, we plot the rest-frame SEDs of 22 Hot DOGs based on the best-fitting with the Torus+GB model. The restframe SEDs have been normalized to the total IR luminosity $L_{\mathrm{IR}}^{\text {tot }}$. Then we derive a median SED by taking the median value of 22 normalized rest-frame SEDs of Hot DOGs. The median SED of the submm-detected Hot DOGs shows several features consistent with previous works (Wu et al. 2012; Jones et al. 2014; Tsai et al. 2015). It has a very steep $1-5 \mu \mathrm{m}$ spectrum, which could be due to the selection effect of Hot DOGs. It becomes rather flat within the wavelength range of $\sim 10-50 \mu \mathrm{m}$ where the torus emission dominates the energy output. Then it sharply drops at $>100 \mu \mathrm{m}$.

We also compare the median SED of the submm-detected Hot DOGs with other known templates from Polletta et al. (2007), including Type 1 QSOs (QSO1), Type 2 QSOs (QSO2), a starburst galaxy, Arp 220, and a heavily obscured AGN-starburst composite, Mrk 231. We find that Hot DOGs have the highest luminosity ratio between mid-IR and submm at rest frame compared to other templates. The relatively weak emission at $>100 \mu \mathrm{m}$ in the Hot DOG median SED may be due to them having higher temperature cold dust, which will also be discussed in Section 5.1. Within the wavelength range of $\sim 6-50 \mu \mathrm{m}$, the median SEDs of Hot DOGs are very similar to those of QSO1 and QSO2. This result supports the argument that Hot DOGs are heavily dust-obscured QSOs.

\section{DISCUSSIONS}

\subsection{The $T_{\text {dust }}-L_{\mathrm{IR}}$ Relation}

The cold dust temperature $T_{\text {dust }}$ for the Hot DOG sample has been derived as described in Section 3 (see also Table 5). We note that the formula of the graybody in Equation (3) is for general opacity. Adopting general opacity, the dust temperatures of Hot DOGs range from 45 to $95 \mathrm{~K}$, with a median value of about $72 \mathrm{~K}$. For some previous studies on SMGs (e.g., Yang et al. 2007; Lapi et al. 2011), the optically thin regime has been

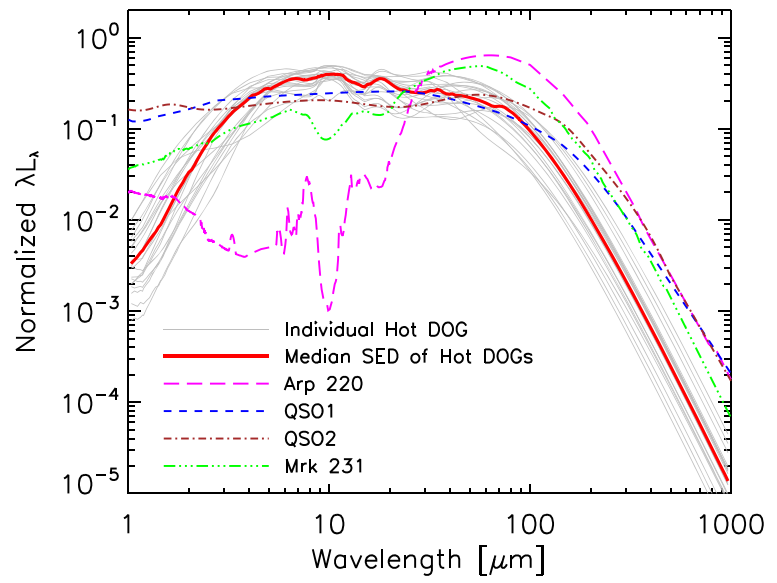

Figure 5. Normalized rest-frame SEDs (gray thin lines) and the median SED (red thick line) of the submm-detected Hot DOGs. The SEDs are based on the best-fitting with the Torus+GB model and normalized to the total IR luminosity $L_{\mathrm{IR}}^{\text {tot }}$. The median SED is derived by taking the median value of all 22 best-fitting SEDs. Individual SEDs and the median SED have been compared to other templates, including Type 1 QSOs (QSO1), Type 2 QSOs (QSO2), a starburst galaxy, Arp 220, and a heavily obscured AGN-starburst composite, Mrk 231 from Polletta et al. (2007).

assumed and the term $\left(1-e^{-\left(\frac{\lambda_{0}}{\lambda}\right)^{\beta}}\right)$ in Equation (3) can be simplified as $\left(\frac{\lambda_{0}}{\lambda}\right)^{\beta}$ at $\lambda \gg \lambda_{0}$. The different assumption on the optical depth results in the differences for the derived dust temperatures. The dust temperatures derived with general opacity are higher than those with the optical thin assumption (Conley et al. 2011; Magdis et al. 2012; Huang et al. 2014). We test how the choice of opacity will affect the estimation of the cold dust temperature. Assuming an optically thin case and using $S_{\lambda} \propto \lambda^{-\beta} B_{\lambda}\left(T_{\text {dust }}\right)$ to describe GB component, we derive the dust temperatures of Hot DOGs ranging from 35 to $74 \mathrm{~K}$ with a median value of about $49 \mathrm{~K}$, which is on average $23 \mathrm{~K}$ lower than that of general opacity. Under the optically thin assumption, the derived dust temperatures remain on average hotter than those found in ULIRGs, SMGs, and DOGs, which mostly range from 20 to $50 \mathrm{~K}$ (Kovács et al. 2006; Magdis et al. 2010; Magnelli et al. 2012; Melbourne et al. 2012). 


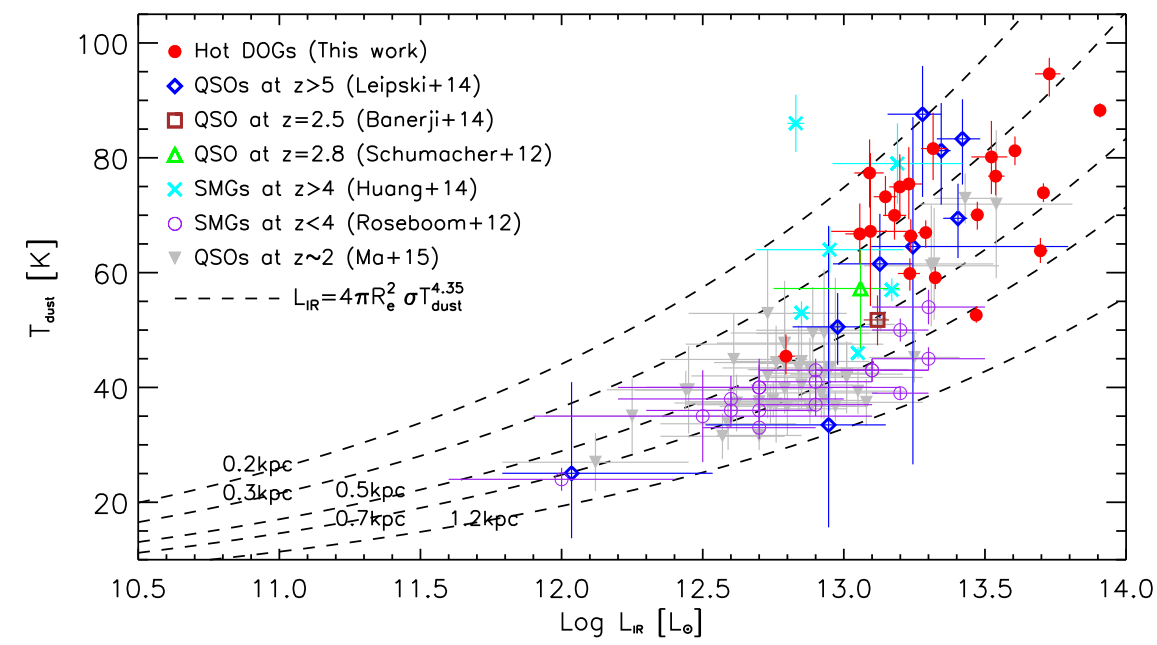

Figure 6. Cold dust temperature as a function of IR luminosity for our Hot DOG sample and other high-redshift populations: SMGs at $z<4$ (Roseboom et al. 2012) and $z>4$ (Huang et al. 2014), QSOs at $z>1.5$ (Ma \& Yan 2015) and $z>5$ (Leipski et al. 2013, 2014), a very red Type 1 QSO ULASJ1234+0907 at $z=2.5$ (Banerji et al. 2014), and a heavily obscured QSO AMS12 at $z=2.8$ (Schumacher et al. 2012). The dashed lines represent the expected $T_{\mathrm{dust}}-L_{\mathrm{IR}}$ relation from Equation (4) with several different $R_{e}$ values $(0.2,0.3,0.5,0.7$, and $1.2 \mathrm{kpc})$.

In Figure 6, we plot the relation between the cold dust temperature $T_{\text {dust }}$ and the IR luminosity of cold dust. We compare our sample with other populations: SMGs at $z<4$ (Roseboom et al. 2012) and $z>4$ (Huang et al. 2014), QSOs at $z>1.5$ (Ma \& Yan 2015) and $z>5$ (Leipski et al. 2013, 2014), a very red Type 1 QSO ULASJ1234+0907 at $z=2.5$ (Banerji et al. 2014), and a heavily obscured QSO AMS12 at $z=2.8$ (Schumacher et al. 2012). As all the compared samples used the graybody with the general opacity form in Equation (3), the comparison between them in Figure 6 should be self-consistent. The adopted parameters of $\beta$ and $\lambda_{0}$ are slightly different for each sample. $\beta=1.8$ and $\lambda_{0}=100 \mu \mathrm{m}$ had been used in SMGs at $z<4$ (Roseboom et al. 2012), while Huang et al. (2014) used $\beta=2.0$ and $\lambda_{0}=100 \mu \mathrm{m}$ for their SMG sample at $z>4$. We selected those $500 \mu \mathrm{m}$ detected $(\sigma>3)$ QSOs with $z>1.5$ from the $250 \mu \mathrm{m}$ detected $(\sigma>5)$ optical-selected QSO sample in Ma \& Yan (2015). They adopted $\beta=1.5$, which is same as the default value in Casey (2012), and $\lambda_{0}=100 \mu \mathrm{m}$ following Draine (2006). For QSOs at $z>5$, we selected nine QSOs with $500 \mu \mathrm{m}$ and/or $1.2 \mathrm{~mm}$ detected from Leipski et al. (2013, 2014). For nine $z>5$ submm $/ \mathrm{mm}$-detected QSOs, the very red Type 1 QSO ULASJ1234+0907 and the heavily obscured QSO AMS12, we re-fitted their IR SEDs with the Torus+GB model, adopting $\beta=1.6$ and $\lambda_{0}=125 \mu \mathrm{m}$, just as we did for our Hot DOG sample.

The locus on the $T_{\text {dust }}-L_{\mathrm{IR}}$ diagram of our Hot DOG sample is consistent with that of submm-detected QSOs in a similar IR luminosity range $\left(L_{\mathrm{IR}}>10^{13} L_{\odot}\right)$. However, compared to SMGs in a similar redshift range (Roseboom et al. 2012), our submm-detected Hot DOGs are more luminous (the median value of $\log L_{\mathrm{IR}}\left[L_{\odot}\right]: 13.3$ versus 12.9) and have hotter dust temperature (the median value of $T_{\text {dust }}: 72 \mathrm{~K}$ versus $40 \mathrm{~K}$ ). Both the red Type 1 QSO ULASJ1234+0907 and the heavily obscured QSO AMS12 seem to follow the $T_{\text {dust }}-L_{\text {IR }}$ relation of our submm-detected Hot DOGs, while they have slightly low temperature and IR luminosities. Interestingly, submm-detected QSOs have the same $T_{\text {dust }}-L_{\mathrm{IR}}$ relation as SMGs in the similar redshift range at $L_{\mathrm{IR}} \leqslant 10^{13} L_{\odot}$, which indicates that they may have similar dust properties.
In order to understand the $T_{\text {dust }}-L_{\mathrm{IR}}$ relation of our submmdetected Hot DOGs and other populations, we try to interpret the observed $T_{\text {dust }}-L_{\mathrm{IR}}$ relation using the Stefan-Boltzmann law following Symeonidis et al. (2013) and Ma \& Yan (2015). We note that the Stefan-Boltzmann law has the form of $L=4 \pi R^{2} \sigma T^{4}$ for a perfect blackbody. While we adopt a graybody in this work, we expect that the $T_{\text {dust }}-L_{\mathrm{IR}}$ relation will have a different form against the perfect blackbody. Following Ma \& Yan (2015), we integrate Equation (3) and find that the $T_{\text {dust }}-L_{\mathrm{IR}}$ relation can be described approximately by the form:

$$
L_{\mathrm{IR}}=4 \pi R_{e}^{2} \sigma T^{\alpha} .
$$

$R_{e}$ can be treated as the effective radius of the equivalent FIRemitting region. We also find that the index $\alpha$ is dependent on the choice of the dust temperature range. For low dust temperature $\left(T_{\text {dust }}<35 \mathrm{~K}\right)$, the index $\alpha$ equals 5.05 , while the value decreases to 4.35 for $T_{\text {dust }} \geqslant 35 \mathrm{~K}$. The value 4.35 of the index $\alpha$ is very close to the adopted value 4.32 in Ma \& Yan (2015). The slight difference of the derived $\alpha$ can rise from the different choices of $\beta$ and $\lambda_{0}$ in Equation (3) between us. As all of our Hot DOGs and most of other populations plotted in Figure 6 have a dust temperature greater than $35 \mathrm{~K}$, we therefore adopt the value 4.35 of the index $\alpha$. We plot the $T_{\text {dust }}-L_{\text {IR }}$ relation expected by Equation (4) with several different $R_{e}(0.2,0.3,0.5,0.7$, and $1.2 \mathrm{kpc}$; see the dashed lines in Figure 6). For Hot DOGs and all other populations plotted in Figure 6 having $L_{\mathrm{IR}}>10^{12} L_{\odot}$, the increase in IR luminosity is mostly due to the increase of the dust temperature. For instance, the $T_{\text {dust }}-L_{\mathrm{IR}}$ relation of SMGs at $z<4$ can be described by Equation (4), adopting $R_{e}=0.7 \mathrm{kpc}$. Compared to SMGs at $z<4$, our Hot DOGs show higher dust temperature, but smaller $R_{e}$ which range from 0.2 to $0.5 \mathrm{kpc}$. Thus the increase in IR luminosity of our Hot DOGs relative to that of SMGs at $z<4$ should be dominated by an increase in dust temperature rather than $R_{e}$. The increase of dust temperature could be due to the more intense radiation field caused by more intense starburst activity and/or buried AGN activity. 


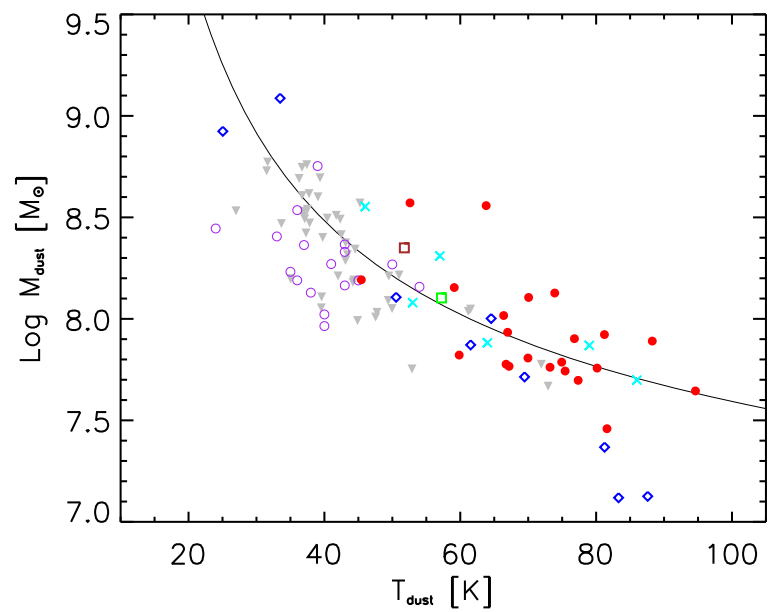

Figure 7. Dust mass $\left(M_{\text {dust }}\right)$ as a function of dust temperature $\left(T_{\text {dust }}\right)$ for our Hot DOG sample and other high-redshift populations. The symbols are the same as in Figure 6. The solid line represents the $M_{\text {dust }}-T_{\text {dust }}$ relation at $z=3.0$ expected by Equation (5), assuming $S_{\nu_{\mathrm{obs}}}=39 \mathrm{mJy}$ at $\nu_{\mathrm{obs}}=600 \mathrm{GHz}$.

\subsection{Dust Mass and Gas Mass}

Our SED fitting with the Torus+GB model decomposes IR emission of Hot DOGs into hot torus and cold dust components. The cold dust temperature has been constrained well. We can therefore estimate the mass of cold dust using:

$$
M_{\text {dust }}=\frac{D_{L}^{2}}{(1+z)} \times \frac{S_{\nu_{\text {obs }}}}{\kappa_{\nu_{\text {rest }}} B\left(\nu_{\text {rest }}, T_{\text {dust }}\right)},
$$

where $D_{L}$ is the luminosity distance, $S_{\nu_{\text {bos }}}$ is the flux density at the observed frequency $\nu_{\mathrm{obs}}, \kappa_{\nu_{\text {rest }}}=\kappa_{0}\left(\nu / \nu_{0}\right)^{\beta}$ is the dust mass absorption coefficient at the rest frequency of the observed band, and $B\left(\nu_{\text {rest }}, T_{\text {dust }}\right)$ is the Planck function at temperature $T_{\text {dust }}$. The main uncertainty of dust mass estimation arises from the choice of the $\kappa_{\nu_{\text {rest }}}$ value. In the literature, the $\kappa_{\nu_{\text {rest }}}$ value can vary by over one order of magnitude at a given frequency/ wavelength: from a very high value of $\kappa_{850 \mu}$ m (i.e., $\kappa_{350 \mathrm{GHz}}$ ) $\sim 11 \mathrm{~cm}^{2} \mathrm{~g}^{-1}$ suggested by laboratory measurements and theoretical modeling, $\kappa_{850 \mu \mathrm{m}} \sim 1.6-8 \mathrm{~cm}^{2} \mathrm{~g}^{-1}$ from the observations of newly formed dust, to a very low value of $\kappa_{850 \mu} \mu \mathrm{m}$ $\left(\sim 0.4 \mathrm{~cm}^{2} \mathrm{~g}^{-1}\right)$, supported by studies of extragalactic systems and diffuse ISM dust in the Galaxy (James et al. 2002; Draine 2003; Dunne et al. 2003; Siebenmorgen et al. 2014). In this paper, we adopt a moderate value of $\kappa_{1 \mathrm{THz}}=20 \mathrm{~cm}^{2} \mathrm{~g}^{-1}$, which is the same as in Wu et al. (2014). Given $\beta=1.6$ and $\kappa_{1 \mathrm{THz}}=20 \mathrm{~cm}^{2} \mathrm{~g}^{-1}$, we can derive $\kappa_{850 \mu m}=3.8 \mathrm{~cm}^{2} \mathrm{~g}^{-1}$. We use the flux density at $500 \mu \mathrm{m}$ (or $850 \mu \mathrm{m}$, if detected) for dust mass estimation of Hot DOGs. For high-redshift SMGs and QSOs, the detected longest-wavelength band (normally among 500,850 or $1200 \mu \mathrm{m}$ ) has been used to estimate their dust masses.

We plot the dust mass as a function of dust temperature for our Hot DOG sample and high-redshift SMGs and QSOs in Figure 7. As we estimate the dust mass of Hot DOGs and all other populations plotted in Figure 7 adopting the same value of $\kappa_{850 \mu \mathrm{m}}$, the dust mass comparison among them will be selfconsistent. The logarithm values of dust mass ( $\left.\log M_{\text {dust }}\left[M_{\odot}\right]\right)$ range from 7.5 to 8.6, with a median value of 7.9 for our Hot DOG sample. The median values of $\log M_{\text {dust }}$ of both SMGs at

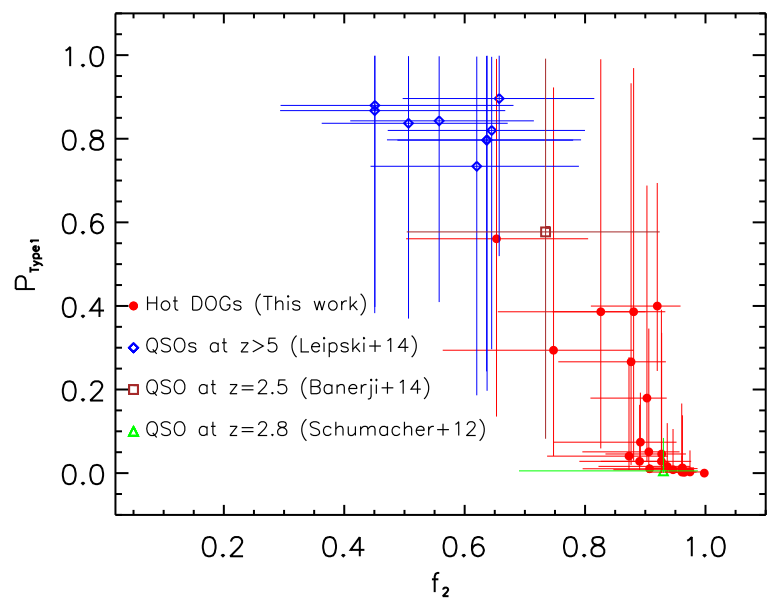

Figure 8. Probability that the object can be observed as a Type 1 AGN $\left(P_{\text {type } 1}\right)$ as a function of the geometrical covering factor of the torus $\left(f_{2}\right)$, which is the ratio between the total torus luminosity and bolometric luminosity $L_{\mathrm{bol}}$.

$z<4$ (purple open circles) and QSOs at $z>1.5$ (gray triangles) are about $0.4-0.5$ dex higher than those of Hot DOGs. Our result is inconsistent with that of Wu et al. (2014). They reported that the cold dust masses of Hot DOGs are comparable to those in submm-detected QSOs with a median value of about $10^{8.5} M_{\odot}$, and a bit higher than those in SMGs. They derived the cold dust masses by assuming a fixed and lower dust temperature $\left(T_{\text {dust }}=35 \mathrm{~K}\right)$. We find that the cold dust masses decrease by a significant factor as the derived dust temperature increases by a factor of about two. In Figure 7, we also plot the $M_{\text {dust }}-T_{\text {dust }}$ relation at $z=3.0$ expected by Equation (5), assuming $S_{\nu_{\mathrm{obs}}}=39 \mathrm{mJy}$ at $\nu_{\mathrm{obs}}=600 \mathrm{GHz}$. For the $M_{\text {dust }}-T_{\text {dust }}$ relation at $z=3.0, M_{\text {dust }} \propto T_{\text {dust }}^{-2.3}$ at $T_{\text {dust }} \geqslant 35 \mathrm{~K}$, while $M_{\text {dust }} \propto T_{\text {dust }}^{-6.6}$ at $T_{\text {dust }}<35 \mathrm{~K}$. The calculation of $M_{\text {dust }}$ can be strongly affected by $T_{\text {dust }}$. As a result, our Hot DOGs with hotter dust temperature have lower dust masses compared to SMGs and submm-detected QSOs, even though they have hyperluminous cold dust emissions $\left(L_{\mathrm{IR}}>\sim 10^{13} L_{\odot}\right)$.

Molecular gas masses in Hot DOGs can be calculated from dust masses, assuming a fiducial dust-to-gas ratio of the Milky Way of $\sim 0.01$. The median value of molecular gas masses in Hot DOGs is about $10^{10} M_{\odot}$. As a comparison, SMGs are more gas-rich than Hot DOGs. Molecular gas masses in SMGs are about $10^{10.5} M_{\odot}$, which are consistent with the estimations by converting $\mathrm{CO} J=1-0$ line luminosity to molecular gas masses with a fiducial CO-to- $\mathrm{H}_{2}$ factor (Bolatto et al. 2013; Carilli \& Walter 2013), see also Figure 3 in Wu et al. 2014). The molecular gas mass in a Hot DOG, W0149+2350, is expected to be $\sim 5.5 \times 10^{9} M_{\odot}$, which is consistent with the non-detection of the CO $J=4-3$ line by CARMA in Wu et al. (2014). Wu et al. (2014) gave a $2 \sigma$ upper limit on molecular gas mass for W0149+2350: $M_{\mathrm{H}_{2}}<3.3 \times 10^{10} M_{\odot}$.

\subsection{The Dust CF}

In Figure 8, we plot the relation between the probability that the object can be observed as a Type $1 \operatorname{AGN}\left(P_{\text {type } 1}\right)$ and the geometrical CF of the torus $\left(f_{2}\right)$ of Hot DOGs and QSOs. We emphasize that both the $P_{\text {type } 1}$ and $f_{2}$ of Hot DOGs and QSOs have been derived from the same SED fitting method with the Torus+GB model, which have been listed in Table 5. As expected by Equation (2), a clear anti-correlation between 


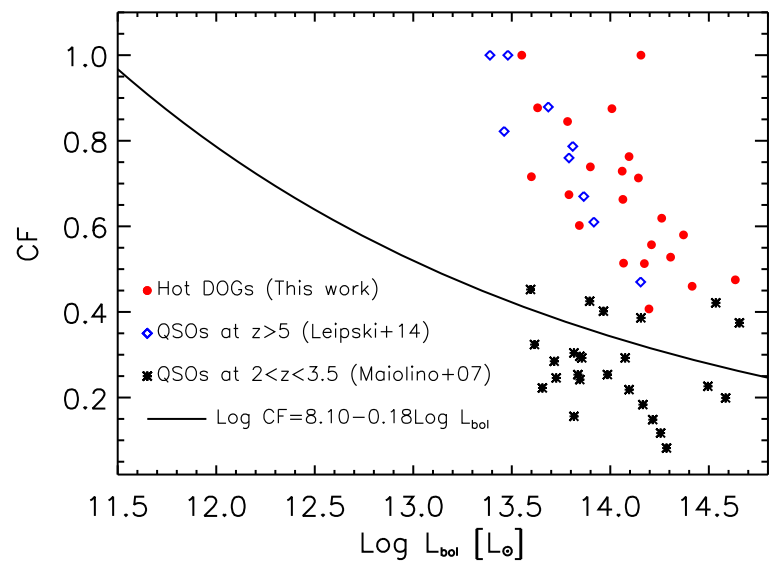

Figure 9. Dust-covering factor (CF) as a function of bolometric luminosity $L_{\mathrm{bol}}$. The solid line represents the CF- $L_{\text {bol }}$ relation derived from Maiolino et al. (2007).

$P_{\text {type } 1}$ and $f_{2}$ has been seen in Figure 8 . As a result of preselection, submm-detected QSOs at $z>5$ (blue diamonds), ULASJ1234+0907 (brown square), and AMS12 (green triangle) have been known as Type 1 QSOs, a Type 1 QSOs with very red color, and a heavily dust-obscured QSO, respectively. Despite the large uncertainties, the derived values of $P_{\text {type } 1}$ and $f_{2}$ are broadly consistent with the known inputs: Type 1 QSOs at $z>5$ having a large value of $P_{\text {type } 1}$ $(\sim 0.8-0.9)$ and a moderate $f_{2}$ value, ULASJ1234+0907 having moderate values of both $P_{\text {type } 1}$ and $f_{2}(\sim 0.6-0.7)$, and AMS12 having $P_{\text {type } 1} \approx 0$ and $f_{2} \approx 1$. The consistency indicates that our SED fitting method is able to recover dust obscuring only based on IR SED. All but one Hot DOGs have $P_{\text {type } 1}<0.4$ and over $2 / 3$ Hot DOGs have $P_{\text {type } 1}<0.1$. All but two Hot DOGs have $f_{2}>0.8$. The low $P_{\text {type } 1 \text { value and high } f_{2}}$ value confirm again that Hot DOGs are heavily dustobscured QSOs.

As mentioned by Mor et al. (2009), the geometrical CF of the torus $\left(f_{2}\right)$, which is the ratio between the total torus luminosity and bolometric luminosity, is different from the apparent $\mathrm{CF}$ of the torus, which is the ratio between the observed luminosity at a given angle and wavelength range and $L_{\text {bol }}$. The apparent $\mathrm{CF}$ can be written as

$$
f(i)=\frac{1}{L_{\mathrm{bol}}} \int_{2 \mu \mathrm{m}}^{100 \mu \mathrm{m}} L_{\lambda} d \lambda
$$

where $L_{\lambda}$ is the rest-frame monochromatic luminosity of the torus. This definition of $f(i)$ is consistent with that of dust $\mathrm{CF}$ defined by Maiolino et al. (2007), where CF is the ratio of thermal infrared emission to the primary AGN radiation. We estimate the $\mathrm{CF}$ values of Hot DOGs by adopting $L_{\text {bol }}=B C \times L_{\mathrm{IR}}^{t}$ in Equation (6), where $B C$ is a bolometric correction factor. Here we adopt $B C=1.4$, as the observed SEDs of Hot DOGs are dominated by IR emission of tori. It is also broadly consistent with the conservative estimations of $L_{\text {bol }}$ in Tsai et al. (2015). The CF values of submm-detected QSOs at $z>5$ have been computed by converting the mid-IRto-optical luminosity ratio (Equation (2) in Maiolino et al. 2007). We plot the relation between the CF values of Hot DOGs, submm-detected QSOs at $z>5$ and $2<z<3.5$ QSOs and their bolometric luminosities in Figure 9. The solid line

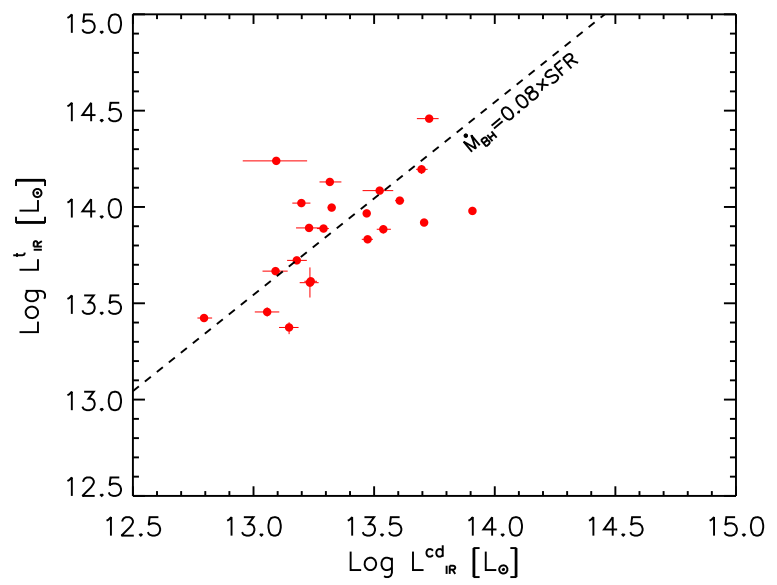

Figure 10. IR luminosity of the cold dust component $L_{\mathrm{IR}}^{\mathrm{cd}}$ vs. the IR luminosity of the torus component $L_{\mathrm{IR}}^{t}$ for Hot DOGs. The dashed line represents the observed $L_{\mathrm{IR}}^{\mathrm{cd}}-L_{\mathrm{IR}}^{t}$ relation of Hot DOGs, which corresponds to the relation between the star formation rate and the black hole growth rate, $\dot{M}_{\mathrm{BH}}=0.08 \times$ SFR (see more details in Section 5.4).

represents the $\mathrm{CF}-L_{\text {bol }}$ relation derived from Maiolino et al. (2007) by combining their Equations (1) and (2). In the literature, whether $\mathrm{CF}$ evolves with redshift remains controversial (e.g., Treister \& Urry 2006; Hasinger 2008; Lusso et al. 2013). However, the trend that CF decreases with increasing bolometric luminosity has been widely found locally and at high redshift (e.g., Hasinger 2008; Treister et al. 2008; Lusso et al. 2013; Ma \& Wang 2013). Our Hot DOGs are similar to submm-detected QSOs at $z>5$ in Leipski et al. (2014), showing a systematic offset from the $\mathrm{CF}-L_{\text {bol }}$ relation with respect to $2<z<3.5$ QSOs in Maiolino et al. (2007). The extremely luminous Hot DOGs $\left(L_{\text {bol }}>10^{13.5} L_{\odot}\right)$ have rather large dust-covering factors $(\mathrm{CF} \sim 0.4-1.0)$, while $2<z<3.5$ QSOs have similar $L_{\mathrm{bol}}$ but much lower values of $\mathrm{CF} \sim 0-0.5$. Thus $\mathrm{CF}$ could span a full range of $0-1$ at $L_{\text {bol }}>10^{13.5} L_{\odot}$. This result may suggest that the previously found anti-correlation between $\mathrm{CF}$ and $L_{\mathrm{bol}}$ could be due to the rare number density of found heavily obscured QSOs at high redshift and the selection bias, which may miss most heavily obscured QSOs in UV/optical and X-ray bands. The recent study on the most luminous AGNs at $z \sim 2-3.5$ by Netzer et al. (2015) found no evidence for a luminosity-dependence of the torus $\mathrm{CF}$, which is consistent with our results.

\subsection{The Coeval Growth of the SMBHs and Their Hosts}

Based on the SED decompositions presented in Section 3, the total IR luminosities of Hot DOGs have been disentangled into the torus and cold dust components. Assuming that the torus and cold dust emissions are related to $\mathrm{SMBH}$ accretion and star formation, respectively, the derived $L_{\mathrm{IR}}^{\mathrm{cd}}-L_{\mathrm{IR}}^{t}$ relation as seen in Figure 10 can be used to investigate the relation between SMBH accretion and star formation. In the following paragraphs, we will describe how we convert $L_{\mathrm{IR}}^{\mathrm{cd}}$ and $L_{\mathrm{IR}}^{t}$ into star formation rates (SFRs) and SMBH growth rate $\left(\dot{M}_{\mathrm{BH}}\right)$, respectively.

We use the simple relation between the SFR and IR luminosity given for local galaxies (Kennicutt 1998), adopting 


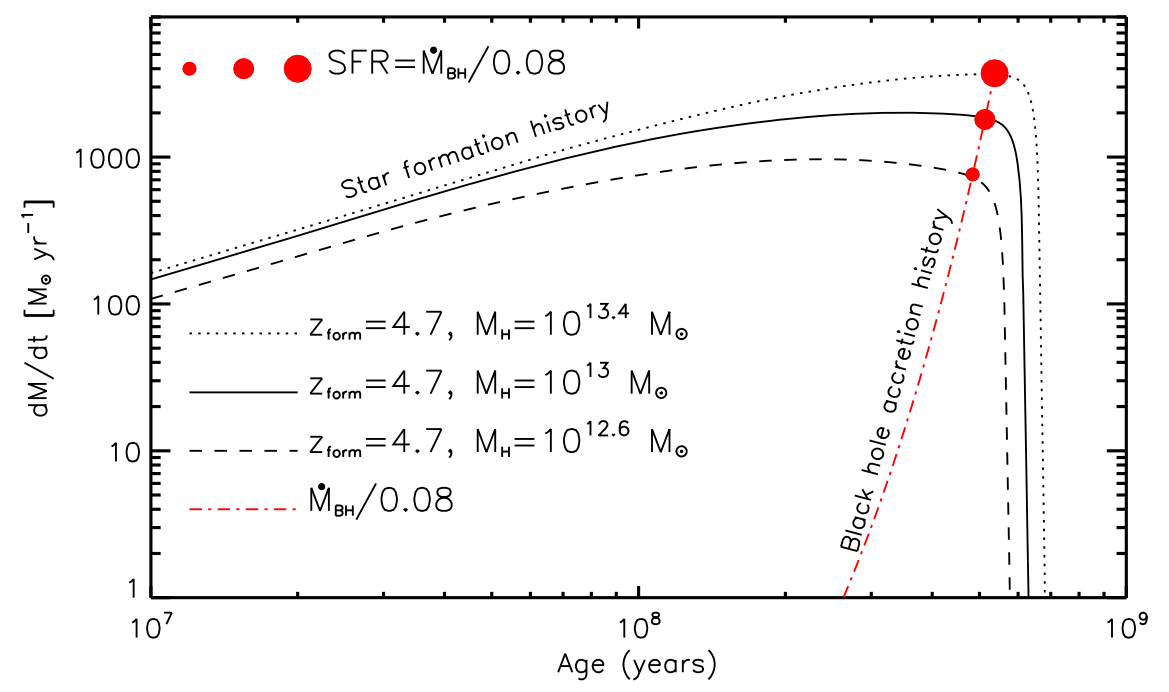

Figure 11. Star formation histories and the black hole growth history predicted by the Granato et al. (2004) model. SFHs are for dark matter halos virilized at formation redshift $z_{\text {form }}=4.7$ with masses $10^{12.60}$ (dashed line), $10^{13.00}$ (solid line), and $10^{13.40} M_{\odot}$ (dotted line), respectively. The dotted-dashed line represents the scaled black hole growth history by multiplying the black hole growth rate, which is described in Equation (10), with a factor of $1 / 0.08$. The filled circles show the moment when SFR $=0.08 \times \dot{M}_{\mathrm{BH}}$ for a given SFH, which is what we have observed in Hot DOGs.

a Chabrier initial mass function (Chabrier 2003):

$$
\frac{\mathrm{SFR}}{M_{\odot} \mathrm{yr}^{-1}}=1.0 \times 10^{-10} \frac{L_{\mathrm{IR}}^{\mathrm{cd}}}{L_{\odot}}
$$

The SFRs of Hot DOGs in our sample span from 600 to $\sim 6000 M_{\odot} \mathrm{yr}^{-1}$, with a median value of $\sim 2000 M_{\odot} \mathrm{yr}^{-1}$. If adopting a Salpeter IMF (Salpeter 1955), the derived SFRs will increase by a factor of 1.72. The SFRs of Hot DOGs are very high, but not rare at high redshift. Other high- $z$ populations have similar SFRs, such as SMGs (e.g., Chapman et al. 2005; Wardlow et al. 2011; Casey et al. 2013; Barger et al. 2014; Swinbank et al. 2014), high- $z$ radio galaxies (e.g., Seymour et al. 2008; Barthel et al. 2012; Rawlings et al. 2013; Drouart et al. 2014), and high- $z$ QSOs (e.g., Wang et al. 2008, 2011; Leipski et al. 2013, 2014; Ma \& Yan 2015).

The QSO bolometric luminosity $L_{\text {bol }}$ can be determined by black hole mass growth rate $\dot{M}_{\mathrm{BH}}$ and radiative efficiency $\eta$, and can also be estimated from the observed IR luminosity $L_{\mathrm{IR}}^{t}$, adopting a bolometric correction factor $B C$ :

$$
L_{\mathrm{bol}}=\frac{\eta \dot{M}_{\mathrm{BH}} c^{2}}{(1-\eta)}=B C \times L_{\mathrm{IR}}^{t}
$$

The radiative efficiency $\eta$ varies from 0.052 for a non-rotating black hole to 0.3 for a fast rotating black hole (e.g., Shapiro 2005). We adopt the more commonly adopted value $\frac{\eta}{1-\eta}=0.1$ (e.g., Yu \& Tremaine 2002; Marconi et al. 2004; Cao \& Li 2008). The bolometric correction factor can vary from 1.4 to 15 for QSOs in the IR band (e.g., Elvis et al. 1994; Marconi et al. 2004; Richards et al. 2006; Hao et al. 2014; Scott \& Stewart 2014). Here we adopt $B C=1.4$ because the observed SEDs of Hot DOGs are dominated by IR emission of tori. It is also broadly consistent with the conservative estimations of $L_{\mathrm{bol}}$ in Tsai et al. (2015).

Combining Equations (7) and (8), we can convert the observed $L_{\mathrm{IR}}^{\mathrm{cd}}-L_{\mathrm{IR}}^{t}$ relation into the SFR $-\dot{M}_{\mathrm{BH}}$. We derive that $\dot{M}_{\mathrm{BH}}=0.08 \times$ SFR (see the dashed line in Figure 10).
Here we attempt to examine if the observed extreme properties of Hot DOGs can be predicted by the model of galaxy formation and evolution. We employ a physical model for the coevolution of QSOs and their hosts proposed by Granato et al. (2004), hereafter G04, to reproduce the observed properties of Hot DOGs. In the G04 model, SFR can be written as

$$
\mathrm{SFR}=\int \frac{d M_{\mathrm{cold}}}{\max \left[t_{\mathrm{cool}}, t_{\mathrm{dyn}}\right]} \approx \frac{M_{\mathrm{cold}}}{t_{\star}},
$$

where $t_{\text {cool }}$ and $t_{\mathrm{dyn}}$ are the cooling time and dynamical time, respectively. $t_{\star}$ is the star formation timescale averaged over the mass distribution. $M_{\text {cold }}$ is the cold gas mass, which is dependent on the virilized dark matter halo $M_{H}$ and the formation redshift $z_{\text {form }}$. The black hole grows according to gas accretion at a given Eddington ratio $\lambda_{\text {Edd }}$ :

$$
\dot{M}_{\mathrm{BH}}=\frac{\lambda_{\mathrm{Edd}} M_{\mathrm{BH}}}{\tau_{\text {Salp }}}
$$

where $\tau_{\text {Salp }}$ is the Salpeter timescale (Salpeter 1964). For the adopted value of $\eta$, where $\frac{\eta}{1-\eta}=0.1, \tau_{\text {Salp }} \sim 50 \mathrm{Myr}$. We assume a seed black hole mass $M_{\mathrm{BH}}^{\mathrm{sed}}=10^{3} M_{\odot}$ and adopt $\lambda_{\mathrm{Edd}}=1.5$. In the G04 model, star formation and black hole growth will be quenched by QSOs and SNe feedback when star formation reaches its peak. More details on the model descriptions and analytical approximations can be found in Granato et al. (2004), Lapi et al. (2006, 2014), Mao et al. (2007), Fan et al. (2008, 2010), and Cai et al. (2013, 2014).

In Figure 11, we plot the model-predicted star formation histories (SFHs) for dark matter halos virilized at formation redshift $z_{\text {form }}=4.7$ with halo masses $10^{12.60}, 10^{13.00}$, and $10^{13.40} M_{\odot}$, respectively. We also plot the scaled black hole growth history by multiplying the black hole growth rate with a factor of $1 / 0.08=12.5$. The filled circles represent the time when SFR $=0.08 \times \dot{M}_{\mathrm{BH}}$ for a given SFH, which is what we have observed in Hot DOGs. The corresponding redshift at that time is about $z \sim 3$, which is close to the median value of redshift distribution in Hot DOGs. At the time marked by the 


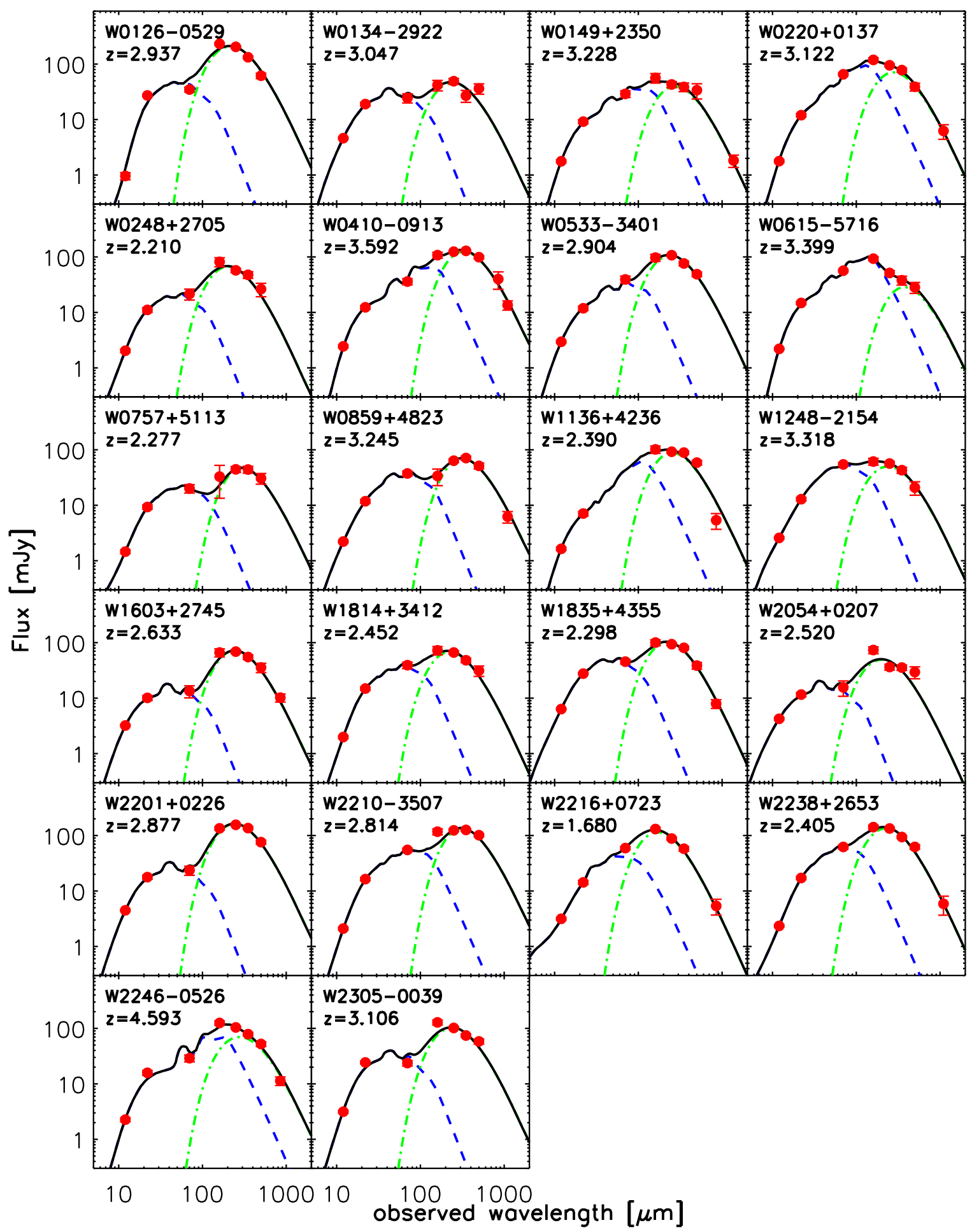

Figure 12. Best-fit (or MAP) model SEDs with the Torus+GB model for 22 Hot DOGs in our sample. The filled circles represent the observed data points. The dashed, dot-dashed, and solid lines are the same as in Figure 1.

filled circles, the model-predicted SFRs are about 700, 2000, and $4500 M_{\odot} \mathrm{yr}^{-1}$ respectively. And black hole masses vary from $\sim 1.0 \times 10^{9} M_{\odot}$ to $\sim 1.0 \times 10^{10} M_{\odot}$. As a comparison, SFRs in Hot DOGs span from 600 to $\sim 6000 M_{\odot} \mathrm{yr}^{-1}$, with a median value of $\sim 2000 M_{\odot} \mathrm{yr}^{-1}$, and black hole masses in Hot DOGs span from $\sim 7.0 \times 10^{8} M_{\odot}$ to $\sim 8.0 \times 10^{9} M_{\odot}$, assuming $\lambda_{\mathrm{Edd}}=1.5$. Both the predicted ranges of SFR and black hole mass are consistent with the observations of Hot DOGs. The
G04 model also predicts that around the peak of SFH the intense star formation will be associated with significant quantities of dust distributed in both AGN tori and hosts, which will bury the central accreting SMBH. This is the probable case in Hot DOGs.

As seen in Figure 11, a simple model can reproduce the observed properties of Hot DOGs. Several probable indications can be deduced from the comparison between the model and 
the observations. (1) Hot DOGs may lie at or close to peaks of both SFH and black hole growth history. (2) Black holes grow exponentially, while star formation has a relatively slow growth rate. Black holes accrete most of their final mass during the last e-folding time. As a consequence, there should be a dusty starburst-dominated phase before the moment when Hot DOGs have been observed. For instance, at $\sim 10^{8}$ year, SFR remains $\sim 1000 M_{\odot} \mathrm{yr}^{-1}$, while $\dot{M}_{\mathrm{BH}}$ would be smaller by $\sim 3$ orders of magnitude (see Figure 11). These are exactly the observed properties of SMGs, which are known as dusty starbursts. Over peaks of both star formation and black hole accretion activities, QSO feedback has been proposed to remove the remaining gas and dust, and then leave an optically bright QSO. Recent work by Diaz-Santos et al. (2015) has drawn the same conclusion, suggesting that one Hot DOG (W2246-0256) is near bursting out the surrounding dust to become an optically bright QSO, based on the study of spatially resolved ALMA [C II] observations. (3) Thus, Hot DOGs may represent a transit phase during the evolution of massive galaxies, transforming galaxies from the dusty starburst-dominated phase to the optically bright QSO phase.

\section{SUMMARY}

In this work, we select 22 submm-detected Hot DOGs with spectroscopic redshift. Their observed IR SEDs have been constructed by combining WISE, Herschel PACS and SPIRE, and SCUBA-2 $850 \mu \mathrm{m}$ data, and other available mm observations. We use a Bayesian SED analysis approach to decompose the observed IR SEDs into two components: torus and cold dust. We use the CLUMPY model to describe torus emission and a graybody to represent the cold dust emission related to star formation. Our main results are summarized below.

1. We compare the Bayesian evidence of the Torus $+\mathrm{GB}$ models with Torus models. We find that the Torus $+\mathrm{GB}$ model has higher Bayesian evidence for all Hot DOGs than the Torus model. We also find that $\ln \left(\frac{\mathrm{ev}_{\text {TORUS }}+\mathrm{GB}}{\mathrm{ev}_{\text {TORUS }}}\right)>10$ (corresponding to odds of $>20000$ : 1), which is strong evidence in favor of the Torus + GB model.

2. Our submm-detected Hot DOGs are all hyperluminous IR galaxies (HyLIRGs, $L_{\mathrm{IR}} \geqslant 10^{13} L_{\odot}$ ) or extremely luminous IR galaxies (ELIRGs, $L_{\mathrm{IR}} \geqslant 10^{14} L_{\odot}$ ). Torus emission dominates the IR energy output. Cold dust emission is on average contributing no more than $\sim 24 \%$ of total IR luminosity, depending on the choice of torus models.

3. We construct a median Hot DOG SED by taking the median value of 22 normalized rest-frame SEDs of Hot DOGs. The median SED is very steep at $1-5 \mu \mathrm{m}$ and becomes rather flat at $\sim 10-50 \mu \mathrm{m}$, then sharply drops at $>100 \mu \mathrm{m}$. Hot DOGs have the highest luminosity ratio between mid-IR and submm at rest frame compared to QSOs and starburst templates. The similarity between Hot DOGs and QSO SEDs at $\sim 10-50 \mu \mathrm{m}$ suggests that the heating sources of Hot DOGs should be buried AGNs.

4. Hot DOGs have high dust temperatures $\left(\left\langle T_{\text {dust }}\right\rangle \sim 72 \mathrm{~K}\right)$ and high IR luminosities of cold dust $L_{\mathrm{IR}}^{\mathrm{cd}}$. Compared to high- $z$ SMGs and QSOs with similar $L_{\mathrm{IR}}^{\mathrm{cd}}$, Hot DOGs have a similar $T_{\text {dust }}-L_{\mathrm{IR}}$ relation. We use the form $L_{\mathrm{IR}}=4 \pi R_{e}^{2} \sigma T^{4.35}$ to describe the expected $T_{\mathrm{dust}}-L_{\mathrm{IR}}$ relation of a graybody at $T_{\text {dust }}>35 \mathrm{~K}$. We find that, at
$L_{\mathrm{IR}}^{\mathrm{cd}}>10^{12} L_{\odot}$, the increase in IR luminosity is mostly due to the increase of dust temperature, rather than dust mass. Compared to SMGs at $z<4$, our Hot DOGs show higher dust temperature, but smaller $R_{e}$. Thus the increase in IR luminosities of our Hot DOGs relative to those of SMGs within a similar redshift range should be dominated by an increase in dust temperature rather than $R_{e}$. The increase of dust temperature could be due to the more intense radiation field caused by more intense starburst activity and/or buried AGN activity.

5. The dust masses of Hot DOGs ( $\left.\log M_{\text {dust }}\left[M_{\odot}\right]\right)$ range from 7.5 to 8.6 , with a median value of 7.9 , which is about 0.4-0.5 dex lower than the values for both SMGs and QSOs within a similar redshift range. The lower dust masses in Hot DOGs are mainly due to the high dust temperature, as the dust mass estimation is strongly affected by $T_{\text {dust }}$ with $M_{\text {dust }} \propto T_{\text {dust }}^{-2.3}$ at $T_{\text {dust }} \geqslant 35 \mathrm{~K}$. The lower dust masses in Hot DOGs will predict lower molecular gas masses. This is consistent with the nondetection of the CO $J=4-3$ line by CARMA in Wu et al. (2014). We will use the deep CO line observations with ALMA to examine this argument during ALMA Cycle 3 (PI: L. Fan).

6. The dust CF of Hot DOGs spans from 0.4 to 1.0, which deviates from the trend that the dust CF decreases with increasing bolometric luminosity. Hot DOGs have heavy dust obscuration and high bolometric luminosity, which could have been missed in the previous UV/optical and X-ray AGN samples.

7. We investigate the possible evolutionary path of Hot DOGs by employing a simple physical model. By comparing the model predictions and the observed properties, we suggest that Hot DOGs may lie at or close to both peaks of star formation and black hole growth histories, and represent a transit phase during the evolution of massive galaxies, transforming them from the dusty starburst-dominated phase to the optically bright QSO phase.

This work is supported by the National Natural Science Foundation of China (NSFC, Nos. 11203023, 11303084, and 11433005), the Fundamental Research Funds for the Central Universities (WK3440000001). L.F. acknowledges the support by the Qilu Young Researcher Project of Shandong University. L.F. and K.K. acknowledge the Knut and Alice Wallenberg Foundation for support. Y.H. thanks the support from the Western Light Youth Project. R.N. acknowledges support by FONDECYT grant No. 3140436.

Facilities: WISE, Herschel (PACS, SPIRE), JCMT (SCUBA-2).

\section{APPENDIX A BAYESIAN APPROACH FOR SED FITTING}

BayeSED (Han \& Han 2012, 2014) ${ }^{10}$ is designed to be a general purpose Bayesian SED fitting code, which means that it can be used to fit the multi-wavelength SEDs of galaxies with a combination of whatever SED models. Given any model SED library, which could be too large to be practically used, we first employ principal component analysis (PCA) to reduce the library dimensionality without sacrificing much accuracy.

\footnotetext{
10 https://bitbucket.org/hanyk/bayesed/
} 
Then, a supervised machine learning method, such as the artificial neural network (ANN) algorithm, or K-Nearest Neighbors (KNN) searching, is employed to approximately generate the model SED at any position of the parameter space spanning the model SED library. So, using these methods, the original SED model, which is given as a SED library, can be approximately and very efficiently evaluated at any position of its parameter space. Thanks to these efficient machine learning methods, we can break through the main bottleneck in Bayesian SED fitting (Kauffmann et al. 2003; Salim et al. 2007; da Cunha et al. 2008; Noll et al. 2009; Walcher et al. 2011), which often requires a very extensive sampling of a high-dimensional parameter space.

Similar to other Bayesian SED fitting codes (Asensio Ramos \& Ramos Almeida 2009; Acquaviva et al. 2011; Serra et al. 2011; Johnson et al. 2013), we estimate the parameters of SED models using the posterior probability distribution function (PDF) of parameters. Instead of the more traditional Markov Chain Monte Carlo (MCMC) algorithm, we have employed the newly developed multimodal nested sampling algorithm (MultiNest, Feroz \& Hobson 2008; Feroz et al. 2009) to obtain the posterior PDF of parameters. What makes the MultiNest algorithm different from the MCMC algorithm is its ability to calculate the Bayesian evidence of a model and explore a more complicated parameter space with multiple posterior modes and pronounced (curving) degeneracies in moderately high dimensions. This ability is crucial for a more reasonable analysis of very complicated multi-wavelength SEDs of galaxies. When modeling the SEDs of galaxies (Conroy 2013), it is very common for us to have multiple physically reasonable choices. So, it is very necessary to have a valid tool to discriminate between these possible choices. Bayesian evidence (Jeffreys 1998; Jaynes 2003; Gregory 2005), which quantitatively implements the principle of Occam's razor, can be employed as such a tool. According to the principle of Occam's razor, a model will not only be appreciated for a better explanation of observations but also be punished for more complexity.

The Bayesian parameter estimation and model comparison with BayeSED have been demonstrated in Han \& Han (2012) for a sample of hyperluminous infrared galaxiesusing the CLUMPY AGN torus model (Nenkova et al. 2008a, 2008b) and the Starburst model of Siebenmorgen \& Krügel (2007), and in Han \& Han (2014) for a Ks-selected sample of galaxies in the COSMOS/UltraVISTA field using stellar population synthesis models. In Han \& Han (2014) we also presented an extensive test of the reliability of the BayeSED code for SED fitting of galaxies.

\section{APPENDIX B \\ MODEL COMPARSION AND MODEL PARAMETERS}

The natural logarithm $\ln \left(\mathrm{ev}_{\mathrm{TORUS}}\right), \ln \left(\mathrm{ev}_{\mathrm{TORUS}+\mathrm{GB}}\right)$ of the Bayesian evidence for the Torus and Torus $+\mathrm{GB}$ models and the natural logarithm of the Bayes factor $\ln \left(\frac{\mathrm{ev}_{\text {ToRUS }+\mathrm{GB}}}{\mathrm{ev}_{\mathrm{TORUS}}}\right)$ are presented in Table 4.

In Table 5 we also list the median values and $16 \%$ and $84 \%$ quartiles of 7 free parameters $\left(Y, i, q, \sigma, N_{0}, \tau_{V}, T_{\text {dust }}\right)$ and 2 derived quantities $\left(f_{2}, P_{\text {type1 } 1}\right)$ with the best-fitting Torus + GB model.

\section{APPENDIX C SED FITTING}

In Figure 12, we plot the best-fit (or the maximum a posteriori, i.e., MAP) model SEDs, adopting the Torus + GB model for 22 Hot DOGs in our sample. In all cases, the torus component has a dominant contribution to the SED at observed the wavelength shorter than $100 \mu \mathrm{m}$, which roughly corresponds to $<25 \mu \mathrm{m}$ at rest frame for the Hot DOGs at $z \sim 3$, while the graybody component has a significant contribution at $>100 \mu \mathrm{m}$.

\section{REFERENCES}

Acquaviva, V., Gawiser, E., \& Guaita, L. 2011, ApJ, 737, 47 Alexander, D. M., \& Hickox, R. C. 2012, NewAR, 56, 93 Asensio Ramos, A., \& Ramos Almeida, C. 2009, ApJ, 696, 2075

Assef, R. J., Eisenhardt, P. R. M., Stern, D., et al. 2015a, ApJ, 804, 27 Assef, R. J., Walton, D. J., Brightman, M., et al. 2015b, arXiv:1511.05155 Banerji, M., Fabian, A. C., \& McMahon, R. G. 2014, MNRAS, 439, L51 Barger, A. J., Cowie, L. L., Chen, C.-C., et al. 2014, ApJ, 784, 9

Barnes, J. E., \& Hernquist, L. 1992, ARA\&A, 30, 705

Barthel, P., Haas, M., Leipski, C., \& Wilkes, B. 2012, ApJL, 757, L26

Beelen, A., Cox, P., Benford, D. J., et al. 2006, ApJ, 642, 694

Blain, A. W., Smail, I., Ivison, R. J., Kneib, J.-P., \& Frayer, D. T. 2002, PhR, 369,111

Bolatto, A. D., Wolfire, M., \& Leroy, A. K. 2013, ARA\&A, 51, 207

Cai, Z.-Y., Lapi, A., Bressan, A., et al. 2014, ApJ, 785, 65

Cai, Z.-Y., Lapi, A., Xia, J.-Q., et al. 2013, ApJ, 768, 21

Cao, X., \& Li, F. 2008, MNRAS, 390, 561

Carilli, C. L., \& Walter, F. 2013, ARA\&A, 51, 105

Casey, C. M. 2012, MNRAS, 425, 3094

Casey, C. M., Chen, C.-C., Cowie, L. L., et al. 2013, MNRAS, 436, 1919

Casey, C. M., Narayanan, D., \& Cooray, A. 2014, PhR, 541, 45

Chabrier, G. 2003, PASP, 115, 763

Chapman, S. C., Blain, A. W., Smail, I., \& Ivison, R. J. 2005, ApJ, 622, 772

Conley, A., Cooray, A., Vieira, J. D., et al. 2011, ApJL, 732, L35

Conroy, C. 2013, ARA\&A, 51, 393

Cutri, R. M., et al. 2013, yCat, 2328, 0

da Cunha, E., Charlot, S., \& Elbaz, D. 2008, MNRAS, 388, 1595

Dekel, A., Birnboim, Y., Engel, G., et al. 2009, Natur, 457, 451

Dey, A., Soifer, B. T., Desai, V., et al. 2008, ApJ, 677, 943

Diaz-Santos, T., Assef, R. J., Blain, A. W., et al. 2015, arXiv:1511.04079

Draine, B. T. 2003, ARA\&A, 41, 241

Draine, B. T. 2006, ApJ, 636, 1114

Drouart, G., De Breuck, C., Vernet, J., et al. 2014, A\&A, 566, A53

Dunne, L., Eales, S., Ivison, R., Morgan, H., \& Edmunds, M. 2003, Natur, 424, 285

Eisenhardt, P. R. M., Wu, J., Tsai, C.-W., et al. 2012, ApJ, 755, 173

Elvis, M., Wilkes, B. J., McDowell, J. C., et al. 1994, ApJS, 95, 1

Fan, L., Lapi, A., Bressan, A., et al. 2010, ApJ, 718, 1460

Fan, L., Lapi, A., De Zotti, G., \& Danese, L. 2008, ApJL, 689, L101

Feroz, F., \& Hobson, M. P. 2008, MNRAS, 384, 449

Feroz, F., Hobson, M. P., \& Bridges, M. 2009, MNRAS, 398, 1601

Frey, S., Paragi, Z., Gabányi, K. É, \& An, T. 2016, MNRAS, 455, 2058

Granato, G. L., De Zotti, G., Silva, L., Bressan, A., \& Danese, L. 2004, ApJ, 600,580

Gregory, P. 2005, Bayesian Logical Data Analysis for the Physical Sciences (New York: Cambridge Univ. Press)

Griffin, M. J., Abergel, A., Abreu, A., et al. 2010, A\&A, 518, L3

Han, Y., \& Han, Z. 2012, ApJ, 749, 123

Han, Y., \& Han, Z. 2014, ApJS, 215, 2

Hao, H., Elvis, M., Civano, F., et al. 2014, MNRAS, 438, 1288

Hasinger, G. 2008, A\&A, 490, 905

Hopkins, P. F., Cox, T. J., Kereš, D., \& Hernquist, L. 2008, ApJS, 175, 390

Hopkins, P. F., Hernquist, L., Cox, T. J., et al. 2006, ApJS, 163, 1

Huang, J.-S., Rigopoulou, D., Magdis, G., et al. 2014, ApJ, 784, 52

James, A., Dunne, L., Eales, S., \& Edmunds, M. G. 2002, MNRAS, 335, 753

Jaynes, E. T. 2003, Probability Theory: The Logic of Science (UK: Cambridge Univ. Press)

Jeffreys, H. 1961, The Theory of Probability (3rd ed., Oxford: Oxford Univ. Press)

Jeffreys, H. 1998, The Theory of Probability (Oxford: OUP) 
Johnson, S. P., Wilson, G. W., Tang, Y., \& Scott, K. S. 2013, MNRAS, 436, 2535

Jones, S. F., Blain, A. W., Stern, D., et al. 2014, MNRAS, 443, 146

Kauffmann, G., Heckman, T. M., White, S. D. M., et al. 2003, MNRAS, 341,33

Kennicutt, R. C., Jr. 1998, ARA\&A, 36, 189

Kovács, A., Chapman, S. C., Dowell, C. D., et al. 2006, ApJ, 650, 592

Lapi, A., González-Nuevo, J., Fan, L., et al. 2011, ApJ, 742, 24

Lapi, A., Raimundo, S., Aversa, R., et al. 2014, ApJ, 782, 69

Lapi, A., Shankar, F., Mao, J., et al. 2006, ApJ, 650, 42

Leipski, C., Meisenheimer, K., Walter, F., et al. 2013, ApJ, 772, 103

Leipski, C., Meisenheimer, K., Walter, F., et al. 2014, ApJ, 785, 154

Lusso, E., Hennawi, J. F., Comastri, A., et al. 2013, ApJ, 777, 86

Ma, X.-C., \& Wang, T.-G. 2013, MNRAS, 430, 3445

Ma, Z., \& Yan, H. 2015, ApJ, 811, 58

Magdis, G. E., Daddi, E., Béthermin, M., et al. 2012, ApJ, 760, 6

Magdis, G. E., Elbaz, D., Hwang, H. S., et al. 2010, MNRAS, 409, 22

Magnelli, B., Lutz, D., Santini, P., et al. 2012, A\&A, 539, A155

Maiolino, R., Shemmer, O., Imanishi, M., et al. 2007, A\&A, 468, 979

Mao, J., Lapi, A., Granato, G. L., de Zotti, G., \& Danese, L. 2007, ApJ, 667,655

Marconi, A., Risaliti, G., Gilli, R., et al. 2004, MNRAS, 351, 169

Melbourne, J., Soifer, B. T., Desai, V., et al. 2012, AJ, 143, 125

Mor, R., Netzer, H., \& Elitzur, M. 2009, ApJ, 705, 298

Mullaney, J. R., Alexander, D. M., Goulding, A. D., \& Hickox, R. C. 2011, MNRAS, 414, 1082

Nenkova, M., Ivezić, Ž, \& Elitzur, M. 2002, ApJL, 570, L9

Nenkova, M., Sirocky, M. M., Ivezić, Ž, \& Elitzur, M. 2008a, ApJ, 685, 147

Nenkova, M., Sirocky, M. M., Nikutta, R., Ivezić, Ž, \& Elitzur, M. 2008b, ApJ, 685,160

Netzer, H., Lani, C., Nordon, R., et al. 2015, arXiv:1511.07876

Noll, S., Burgarella, D., Giovannoli, E., et al. 2009, A\&A, 507, 1793

Piconcelli, E., Vignali, C., Bianchi, S., et al. 2015, A\&A, 574, L9

Pilbratt, G. L., Riedinger, J. R., Passvogel, T., et al. 2010, A\&A, 518, L1

Poglitsch, A., Waelkens, C., Geis, N., et al. 2010, A\&A, 518, L2

Polletta, M., Tajer, M., Maraschi, L., et al. 2007, ApJ, 663, 81
Rawlings, J. I., Seymour, N., Page, M. J., et al. 2013, MNRAS, 429, 744

Richards, G. T., Lacy, M., Storrie-Lombardi, L. J., et al. 2006, ApJS, 166, 470

Roseboom, I. G., Ivison, R. J., Greve, T. R., et al. 2012, MNRAS, 419, 2758

Salim, S., Rich, R. M., Charlot, S., et al. 2007, ApJS, 173, 267

Salpeter, E. E. 1955, ApJ, 121, 161

Salpeter, E. E. 1964, ApJ, 140, 796

Sanders, D. B., \& Mirabel, I. F. 1996, ARA\&A, 34, 749

Schumacher, H., Martínez-Sansigre, A., Lacy, M., et al. 2012, MNRAS, 423,2132

Scott, A. E., \& Stewart, G. C. 2014, MNRAS, 438, 2253

Serra, P., Amblard, A., Temi, P., et al. 2011, ApJ, 740, 22

Seymour, N., Dwelly, T., Moss, D., et al. 2008, MNRAS, 386, 1695

Shapiro, S. L. 2005, ApJ, 620, 59

Siebenmorgen, R., Heymann, F., \& Efstathiou, A. 2015, A\&A, 583, A120

Siebenmorgen, R., \& Krügel, E. 2007, A\&A, 461, 445

Siebenmorgen, R., Voshchinnikov, N. V., \& Bagnulo, S. 2014, A\&A, 561, A82

Stern, D., Lansbury, G. B., Assef, R. J., et al. 2014, ApJ, 794, 102

Swinbank, A. M., Simpson, J. M., Smail, I., et al. 2014, MNRAS, 438, 1267

Symeonidis, M., Vaccari, M., Berta, S., et al. 2013, MNRAS, 431, 2317

Treister, E., Krolik, J. H., \& Dullemond, C. 2008, ApJ, 679, 140

Treister, E., \& Urry, C. M. 2006, ApJL, 652, L79

Trotta, R. 2008, ConPh, 49, 71

Tsai, C.-W., Eisenhardt, P. R. M., Wu, J., et al. 2015, ApJ, 805, 90

Walcher, J., Groves, B., Budavári, T., \& Dale, D. 2011, Ap\&SS, 331, 1

Wang, R., Wagg, J., Carilli, C. L., et al. 2008, AJ, 135, 1201

Wang, R., Wagg, J., Carilli, C. L., et al. 2011, AJ, 142, 101

Wardlow, J. L., Smail, I., Coppin, K. E. K., et al. 2011, MNRAS, 415, 1479

Wright, E. L., Eisenhardt, P. R. M., Mainzer, A. K., et al. 2010, AJ, 140, 1868

Wu, J., Bussmann, R. S., Tsai, C.-W., et al. 2014, ApJ, 793, 8

Wu, J., Tsai, C.-W., Sayers, J., et al. 2012, ApJ, 756, 96

Wuyts, S., Cox, T. J., Hayward, C. C., et al. 2010, ApJ, 722, 1666

Xu, L., Rieke, G. H., Egami, E., et al. 2015, ApJ, 808, 159

Yang, M., Greve, T. R., Dowell, C. D., \& Borys, C. 2007, ApJ, 660, 1198

Yu, Q., \& Tremaine, S. 2002, MNRAS, 335, 965 